\title{
Benzo-21-Crown-7/Secondary Dialkylammonium Salt [2]Pseudorotaxane- and [2]Rotaxane-Type Threaded Structures
}

Chuanju Zhang, Shijun Li, Jinqiang Zhang, Kelong Zhu, Ning Li, and Feihe Huang* Department of Chemistry, Zhejiang University, Hangzhou 310027, P. R. China. Fax: +86-571-8795-1895; Tel: +86-571-8795-3189; Email address: fhuang@zju.edu.cn.

\section{Supporting Information (26 pages)}

1. Materials and methods

2. Synthesis of hexa(ethylene glycol) ditosylate

3. Synthesis of benzo-21-crown-7

4. Synthesis of secondary dialkylammonium salts 1, 2, 3 and 4

5. Synthesis of 5

S8

6. Synthesis of 6

7. Synthesis of dumbbell-shaped component 7

8. Synthesis of rotaxane 8

9. Partial proton NMR spectra of dumbbell-shaped component 7, rotaxane 8 and rotaxane 8 neutralized by $\mathrm{Et}_{3} N$.

10. X-ray analysis data of rotaxane 8

11. ${ }^{1} H$ NMR spectrum of an equimolar acetone solution of B18C6 and 1

12. ${ }^{1} H$ NMR spectrum of an equimolar acetone solution of B21C7 and 4

13. ${ }^{1} H$ NMR spectrum and ESI-MS of an equimolar acetone solution of B21C7 and 1

14. ${ }^{1} H$ NMR spectrum and ESI-MS of an equimolar acetone solution of B21C7 and 2

15. ${ }^{1}$ H NMR spectrum and ESI-MS of an equimolar acetone solution of B21C7 and 3

16. Determination of association constants of DB24C8・1, DB24C8・2, and DB24C8•3 from chemical shift changes. 

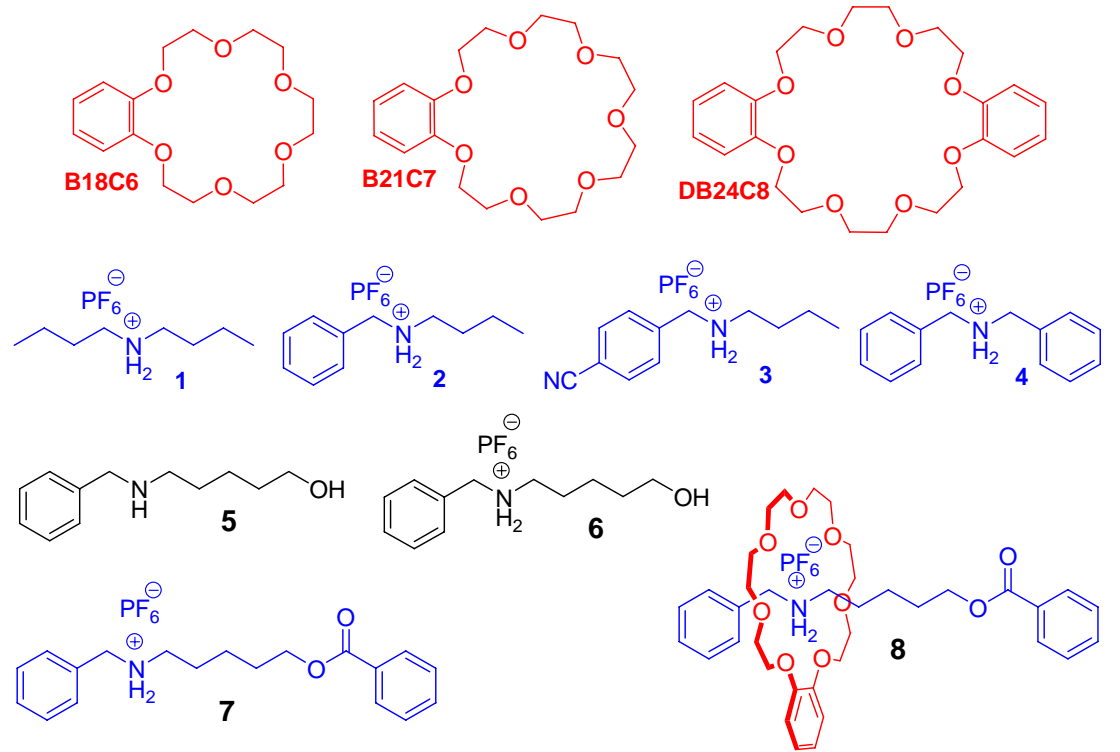

\section{Materials and methods}

Benzo-18-crown-6 (B18C6) and dibenzo-24-crown-8 (DB24C8) were purchased from Acros. Benzo-21-crown-7 (B21C7), ${ }^{\mathrm{S} 1}$ secondary ammonium salts $\mathbf{1},{ }^{\mathrm{S} 2} \mathbf{2}^{\mathrm{S} 2} \mathbf{3}^{\mathrm{S} 3}$ and $4^{\mathrm{S} 2}$ were all synthesized according to literature procedures. Solvents were either employed as purchased or dried according to procedures described in the literature. ${ }^{1} \mathrm{H}$ NMR spectra were collected on a temperature-controlled $400 \mathrm{MHz}$ or $500 \mathrm{MHz}$ spectrometer. 


\section{Synthesis of hexa(ethylene glycol) ditosylate}

Triethylamine $(2.45 \mathrm{~mL}, 17.8 \mathrm{mmol})$ in $\mathrm{CH}_{2} \mathrm{Cl}_{2}(10 \mathrm{~mL})$ was added dropwise to a solution of $p$-toluenesulfonyl chloride ( $8.10 \mathrm{~g}, 42.5 \mathrm{mmol})$ and hexa(ethylene glycol) (5.00 g, $17.7 \mathrm{mmol})$ in $\mathrm{CH}_{2} \mathrm{Cl}_{2}(30 \mathrm{~mL})$ at $0{ }^{\circ} \mathrm{C}$. The suspension was stirred for $2 \mathrm{~h}$ at $0{ }^{\circ} \mathrm{C}$ and then at room temperature for another $16 \mathrm{~h}$. The organic solution was washed twice using $50 \mathrm{~mL}$ of $0.5 \mathrm{M} \mathrm{HCl}$ and $50 \mathrm{~mL}$ of saturated solution of $\mathrm{NaCl}$ and then dried with $\mathrm{Na}_{2} \mathrm{SO}_{4}$. The organic solvent was removed under reduced pressure and the residue was purified by silica gel flash column chromatography (petroleum ether/ethyl acetate, $v / v 3: 2 \rightarrow$ ethyl acetate) to provide hexa(ethylene glycol) ditosylate $(7.60 \mathrm{~g}$, $72.4 \%$ ) as a yellow oil. The proton NMR spectrum of hexa(ethylene glycol) ditosylate is shown in Figure S1. ${ }^{1} \mathrm{H}$ NMR $\left(400 \mathrm{MHz}, \mathrm{CDCl}_{3}, 22{ }^{\circ} \mathrm{C}\right) \delta(\mathrm{ppm}): 7.79(4 \mathrm{H}, \mathrm{d}, J=$ $8.2 \mathrm{~Hz}), 7.34(4 \mathrm{H}, \mathrm{d}, J=8.2 \mathrm{~Hz}), 4.15(4 \mathrm{H}, \mathrm{t}, J=4.8 \mathrm{~Hz}), 3.68(4 \mathrm{H}, \mathrm{t}, J=4.8 \mathrm{~Hz})$, 3.58-3.61 (16H, m), $2.44(6 \mathrm{H}, \mathrm{s})$.
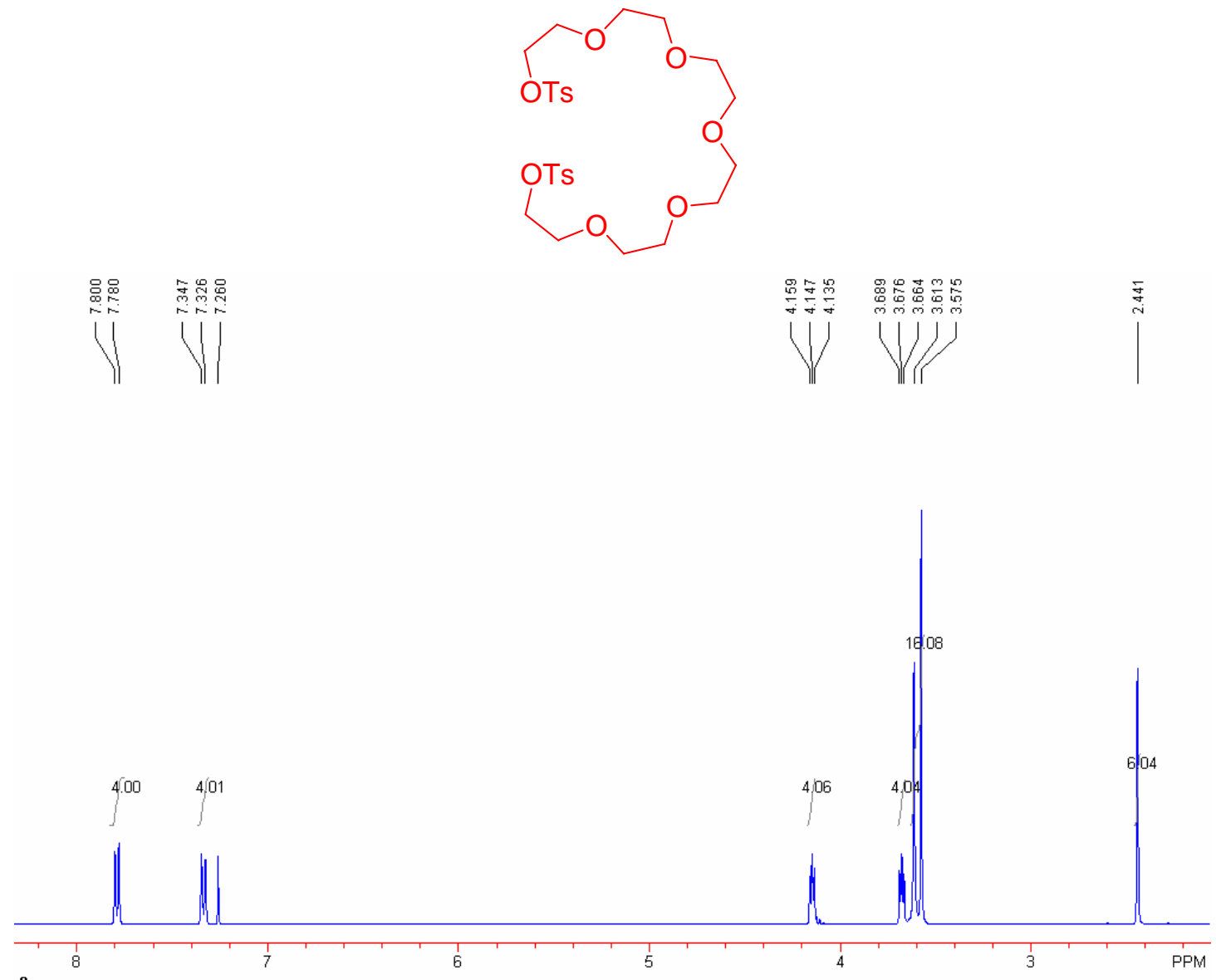

Figure S1. ${ }^{1} \mathrm{H}$ NMR spectrum $\left(400 \mathrm{MHz}, \mathrm{CDCl}_{3}, 22{ }^{\circ} \mathrm{C}\right)$ of hexa(ethylene glycol) ditosylate. 


\section{Synthesis of benzo-21-crown-7}

A mixture of catechol $(1.42 \mathrm{~g}, 12.9 \mathrm{mmol}), \mathrm{K}_{2} \mathrm{CO}_{3}(5.34 \mathrm{~g}, 38.7 \mathrm{mmol}), \mathrm{KPF}_{6}(3.60$ $\mathrm{g}, 19.4 \mathrm{mmol})$ and hexa(ethylene glycol) ditosylate $(7.60 \mathrm{~g}, 12.9 \mathrm{mmol})$ in $100 \mathrm{~mL}$ of $\mathrm{CH}_{3} \mathrm{CN}$ was stirred and refluxed for three days under nitrogen gas protection. After cooling, the mixture was filtered and $\mathrm{CH}_{3} \mathrm{CN}$ was removed with a rotaevaporator, and then $\mathrm{CH}_{2} \mathrm{Cl}_{2}$ was added. After washing with water several times, the organic phase was dried with $\mathrm{Na}_{2} \mathrm{SO}_{4}$ and then concentrated. The residue was purified by silica gel flash column chromatography (petroleum ether/ethyl acetate, $v / v 1: 3$ ) to provide benzo-21-crown-7 $(2.74 \mathrm{~g}, 69 \%)$ as a white solid. mp $134-135{ }^{\circ} \mathrm{C}$. The proton NMR spectrum of the pure benzo-21-crown-7 is shown in Figure S2. ${ }^{1} \mathrm{H}$ NMR (400 MHz, $\left.\mathrm{CDCl}_{3}, 22{ }^{\circ} \mathrm{C}\right) \delta(\mathrm{ppm}): 6.88-6.93(4 \mathrm{H}, \mathrm{m}), 4.17(4 \mathrm{H}, \mathrm{t}, J=4.4 \mathrm{~Hz}), 3.92(4 \mathrm{H}, \mathrm{t}, J=$ $4.4 \mathrm{~Hz}), 3.78-3.81(4 \mathrm{H}, \mathrm{m}), 3.72-3.75(4 \mathrm{H}, \mathrm{m}), 3.66-3.67(8 \mathrm{H}, \mathrm{m})$.
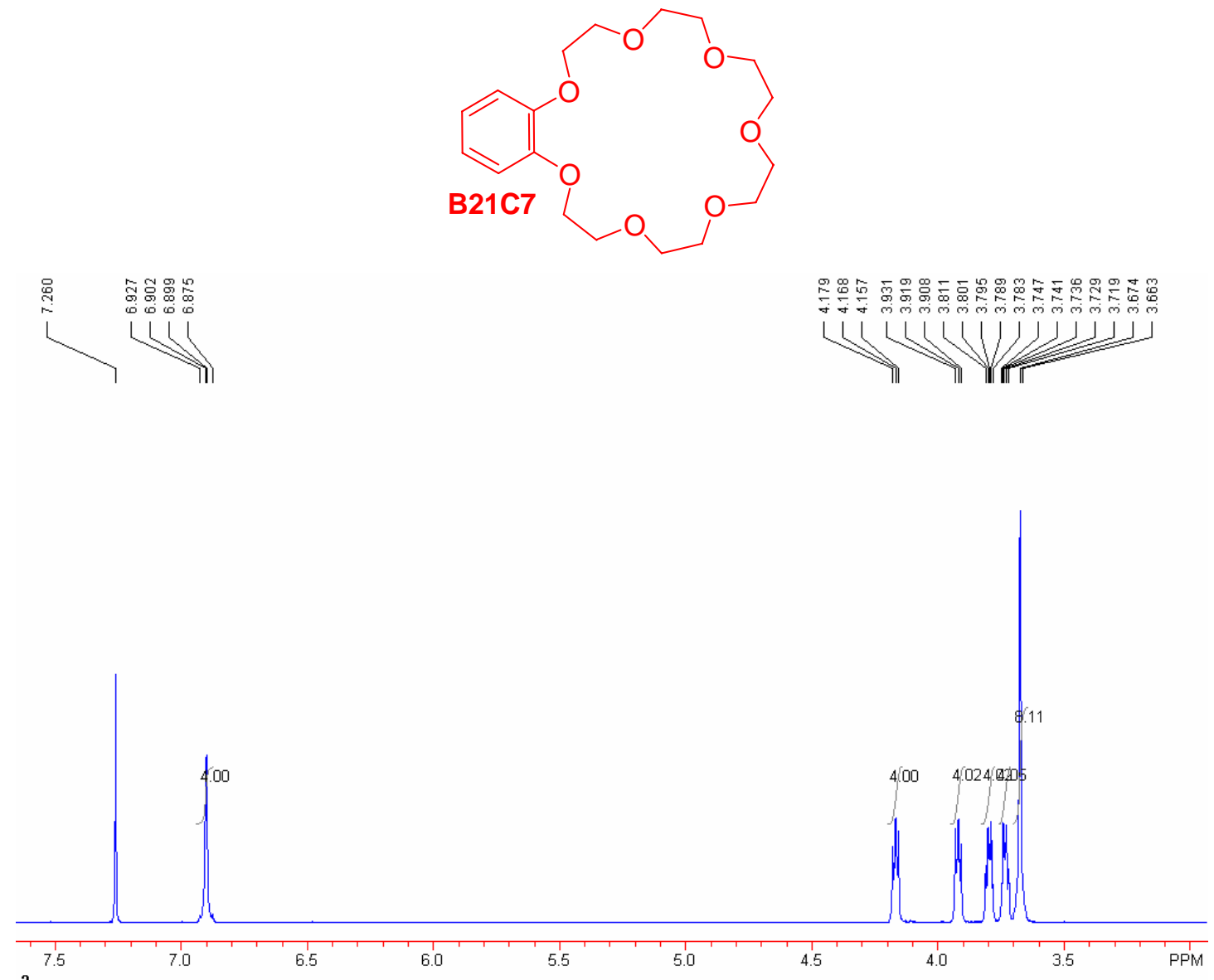

Figure S2. ${ }^{1} \mathrm{H}$ NMR spectrum $\left(400 \mathrm{MHz}, \mathrm{CDCl}_{3}, 22{ }^{\circ} \mathrm{C}\right)$ of benzo-21-crown-7. 


\section{Synthesis of secondary dialkylammonium salts $1,2,3$ and 4}

A solution of butylamine $(10.0 \mathrm{~mL}, 0.100 \mathrm{~mol})$ and $N$-butyraldehyde $(9.00 \mathrm{~mL}$, $0.100 \mathrm{~mol})$ was heated under reflux overnight in $\mathrm{MeOH}(150 \mathrm{~mL})$. After the reaction mixture was cooled to ambient temperature, $\mathrm{NaBH}_{4}(5.67 \mathrm{~g}, 0.15 \mathrm{~mol}$ ) was added portionwise to the stirring solution over a period of $0.5 \mathrm{~h}$. Stirring was maintained under ambient conditions for a further $24 \mathrm{~h}$, after which time $5 \mathrm{M} \mathrm{HCl}$ was added to neutralize excess $\mathrm{NaBH}_{4}$. The mixture was filtered and $\mathrm{MeOH}$ was removed with a rotaevaporator. The residue was extracted with ethyl acetate and the extract was concentrated to get a yellow oil. After the oil was added to a hydrochloric acid solution and stirred for a moment, a white precipitate formed. The mixture was filtered and the solid was dissolved in water to get a saturated solution. The solution was added to a saturated $\mathrm{NH}_{4} \mathrm{PF}_{6}$ solution to produce a precipitate. It was collected by suction filtration and recrystallized from deionized water three times to afford 1 (4.20 $\mathrm{g}, 15.3 \%$ ) as a white solid. mp $230-233{ }^{\circ} \mathrm{C}$ (lit. ${ }^{\text {S2a }} 187-188^{\circ} \mathrm{C}$ ). The proton NMR spectrum of 1 is shown in Figure S3. ${ }^{1} \mathrm{H}$ NMR $\left(400 \mathrm{MHz}, \mathrm{CD}_{3} \mathrm{COCD}_{3}, 22{ }^{\circ} \mathrm{C}\right) \delta$ (ppm): 3.35 (4H, t, $J=7.8 \mathrm{~Hz}), 1.78-1.86(4 \mathrm{H}, \mathrm{m}), 1.41-1.51(4 \mathrm{H}, \mathrm{m}), 0.94(6 \mathrm{H}, \mathrm{t}, J$ $=7.4 \mathrm{~Hz}$ ). The methods for preparing 2, 3 and 4 are the same. $\mathrm{mp}$ of 2: $221-223{ }^{\circ} \mathrm{C}$ (lit. ${ }^{\text {S2a }} 155-158{ }^{\circ} \mathrm{C}$ ). Yield: $25.3 \%$. The proton NMR spectrum of $\mathbf{2}$ is shown in Figure S4. ${ }^{1} \mathrm{H}$ NMR $\left(400 \mathrm{MHz}, \mathrm{CD}_{3} \mathrm{COCD}_{3}, 22{ }^{\circ} \mathrm{C}\right) \delta(\mathrm{ppm}): 8.00-8.30(2 \mathrm{H}, \mathrm{m}), 7.58-7.60$ $(2 \mathrm{H}, \mathrm{m}), 7.46-7.49(3 \mathrm{H}, \mathrm{m}), 4.58(2 \mathrm{H}, \mathrm{s}), 3.41-3.45(2 \mathrm{H}, \mathrm{m}), 1.83-1.91(2 \mathrm{H}, \mathrm{m})$, $1.42-1.52(2 \mathrm{H}, \mathrm{m}), 0.92-0.96(3 \mathrm{H}, \mathrm{m}) . \mathrm{mp}$ of $3: 209-210{ }^{\circ} \mathrm{C}$. Yield: $29.0 \%$. The proton and carbon NMR spectra of 3 are shown in Figures S5 and S6. ${ }^{1} \mathrm{H}$ NMR (500 $\left.\mathrm{MHz}, \mathrm{CD}_{3} \mathrm{COCD}_{3}, 22{ }^{\circ} \mathrm{C}\right) \delta(\mathrm{ppm}): 7.84-7.91(4 \mathrm{H}, \mathrm{m}), 4.73(2 \mathrm{H}, \mathrm{s}), 3.49(2 \mathrm{H}, \mathrm{t}, J$ $=7.5 \mathrm{~Hz}), 1.87-1.90(2 \mathrm{H}, \mathrm{m}), 1.46-1.50(2 \mathrm{H}, \mathrm{m}), 0.95(3 \mathrm{H}, \mathrm{t}, J=7.5 \mathrm{~Hz}) .{ }^{13} \mathrm{C}(100$ $\left.\mathrm{MHz}, \mathrm{CD}_{3} \mathrm{COCD}_{3}, 22{ }^{\circ} \mathrm{C}\right) \delta(\mathrm{ppm}): 136.2,132.5,130.9,117.7,113.0,51.0,48.3,27.7$, 19.1, 12.6. HRMS: $\mathrm{m} / \mathrm{z}$ calcd for $\left[\mathrm{M}-\mathrm{PF}_{6}\right]^{+} \mathrm{C}_{20} \mathrm{H}_{17} \mathrm{~N}_{2}, 189.1386$, found 189.1378, error 4.2 ppm. mp of 4: $209-210{ }^{\circ} \mathrm{C}$ (lit. ${ }^{\text {S2a }} 205-208{ }^{\circ} \mathrm{C}$ ). Yield: $34.5 \%$. The proton NMR spectrum of 4 is shown in Figure S7. ${ }^{1} \mathrm{H}$ NMR $\left(500 \mathrm{MHz}, \mathrm{CD}_{3} \mathrm{COCD}_{3}, 22{ }^{\circ} \mathrm{C}\right) \delta$ (ppm): 7.59-7.61 (4H, m), 7.47-7.49 (6H, m), 4.67 (4H, s). 

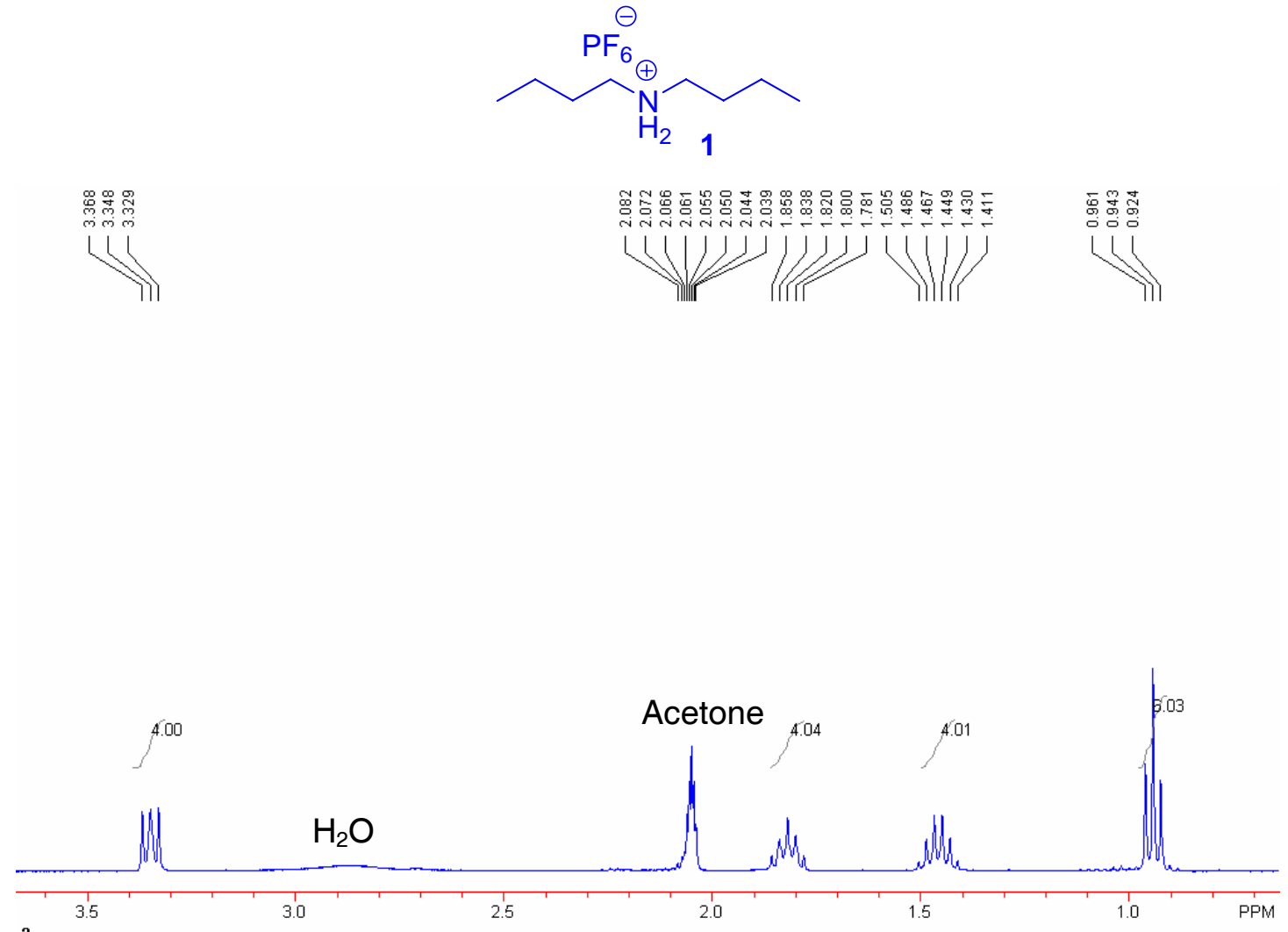

Figure S3. ${ }^{1} \mathrm{H}$ NMR spectrum $\left(400 \mathrm{MHz}, \mathrm{CD}_{3} \mathrm{COCD}_{3}, 22{ }^{\circ} \mathrm{C}\right)$ of $\mathbf{1}$.

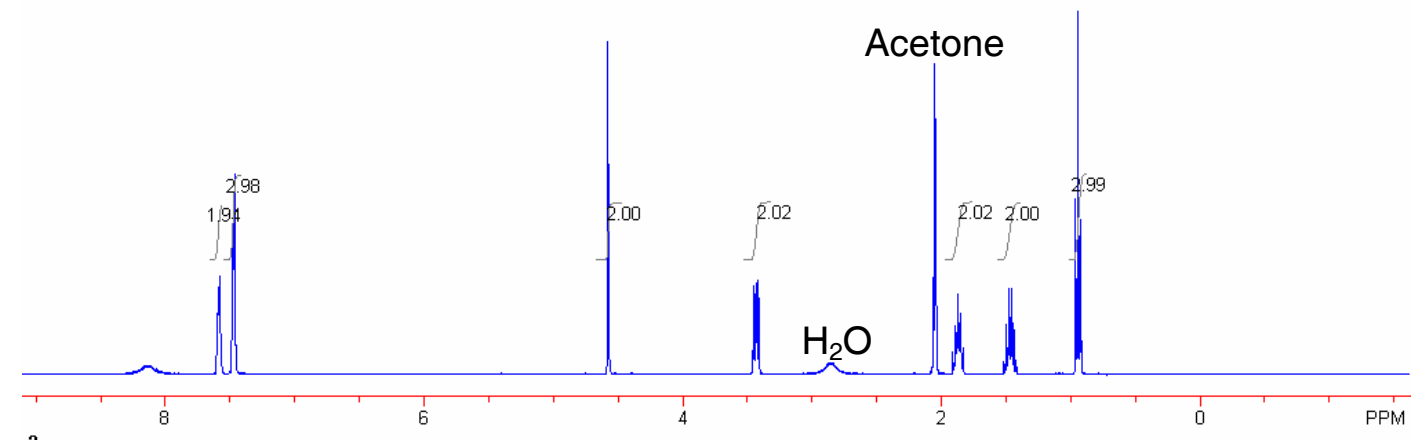

Figure S4. ${ }^{1} \mathrm{H}$ NMR spectrum $\left(400 \mathrm{MHz}, \mathrm{CD}_{3} \mathrm{COCD}_{3}, 22^{\circ} \mathrm{C}\right)$ of 2. 


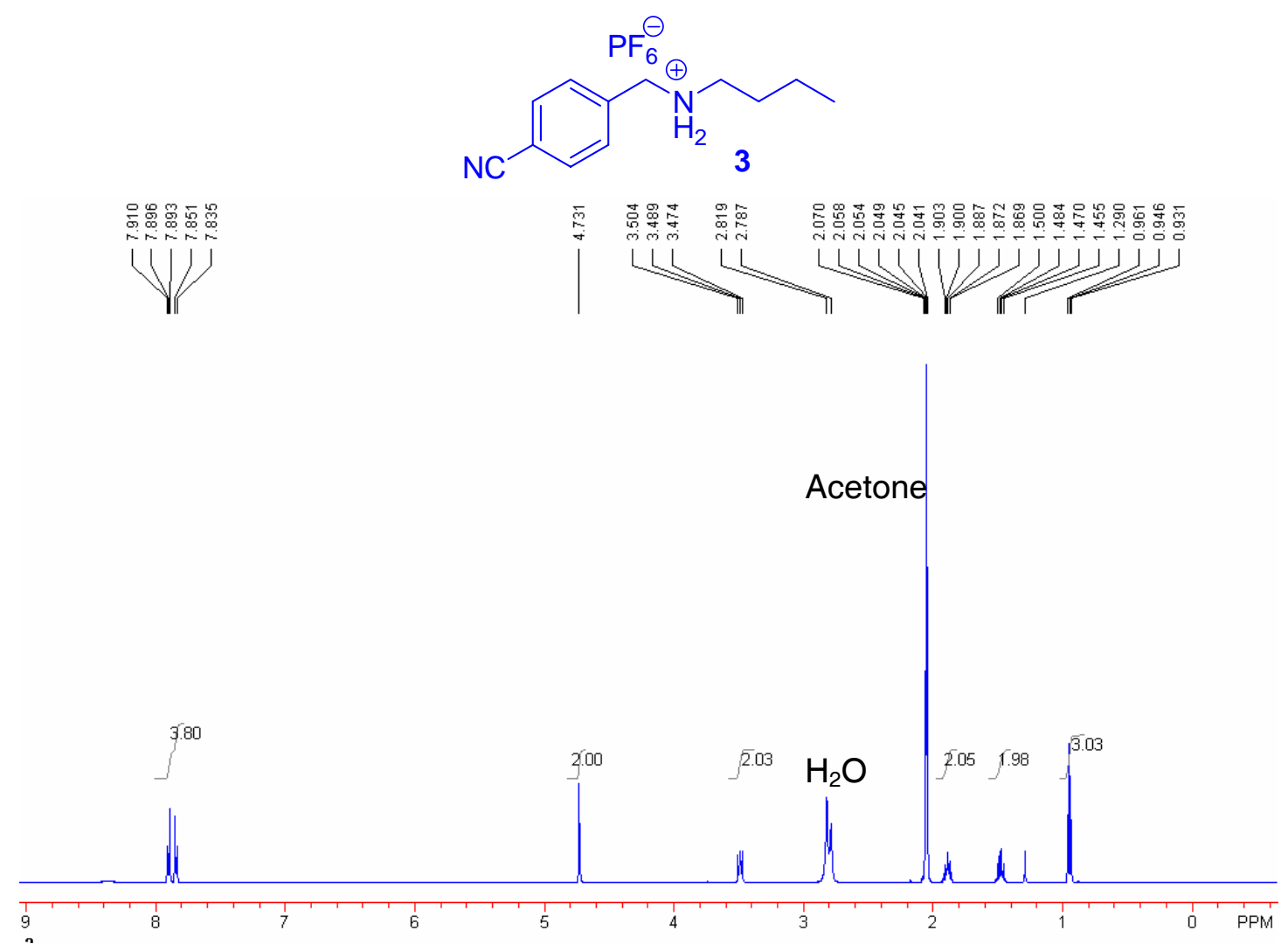

Figure S5. ${ }^{1} \mathrm{H}$ NMR spectrum $\left(500 \mathrm{MHz}, \mathrm{CD}_{3} \mathrm{COCD}_{3}, 22{ }^{\circ} \mathrm{C}\right)$ of 3 .

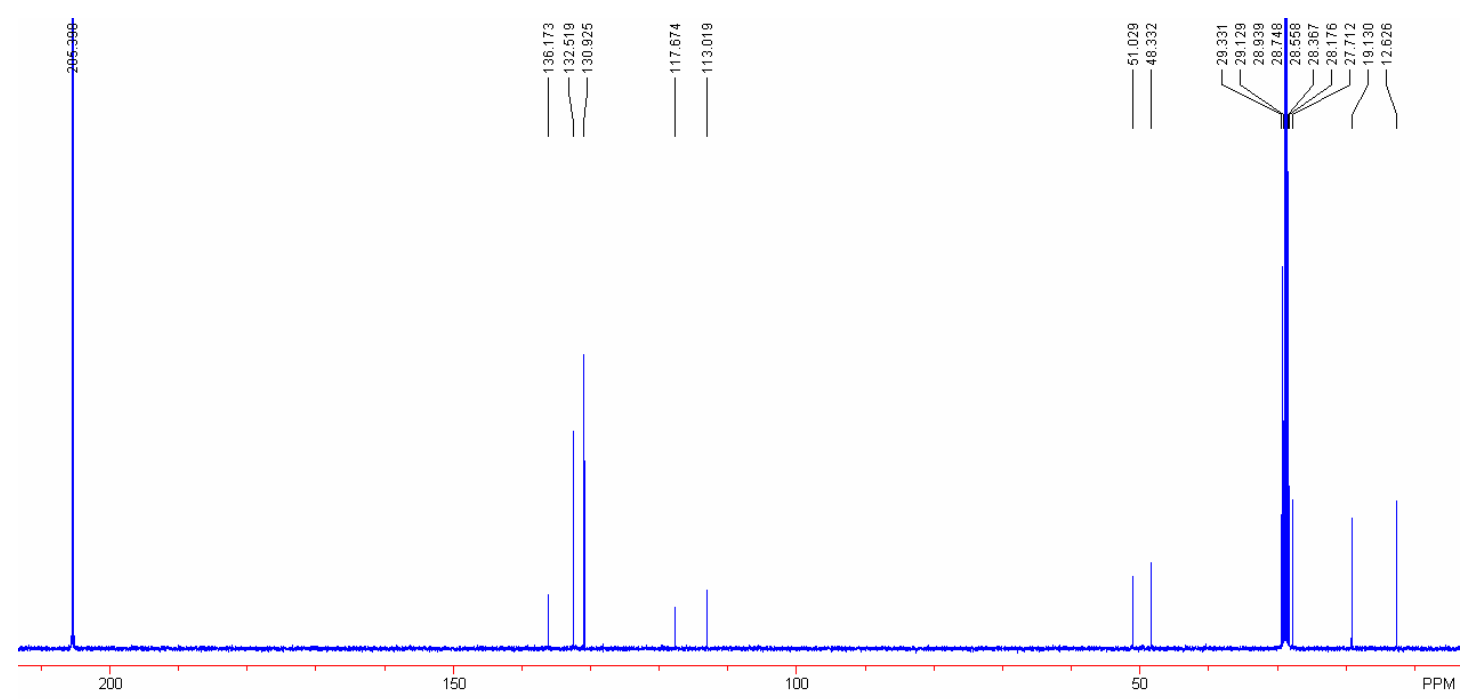

Figure S6. ${ }^{13} \mathrm{C}$ NMR spectrum $\left(100 \mathrm{MHz}, \mathrm{CD}_{3} \mathrm{COCD}_{3}, 22{ }^{\circ} \mathrm{C}\right)$ of 3 . 


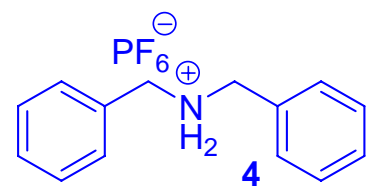

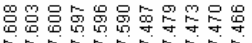

(1) 1) 1) 1)
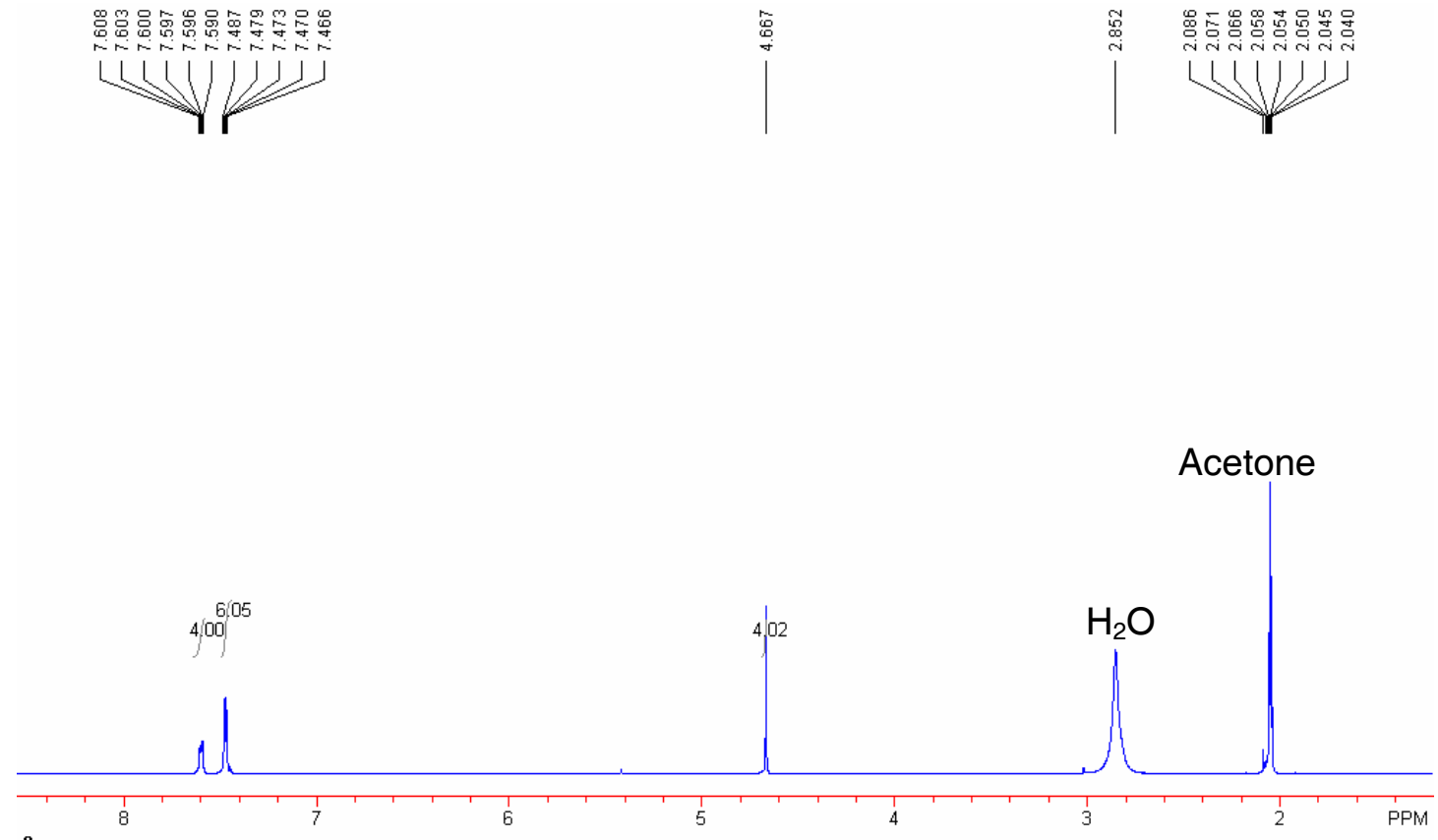

Figure S7. ${ }^{1} \mathrm{H}$ NMR spectrum $\left(500 \mathrm{MHz}, \mathrm{CD}_{3} \mathrm{COCD}_{3}, 22^{\circ} \mathrm{C}\right)$ of 4 .

\section{Synthesis of 5}

A solution of benzaldehyde $(5.60 \mathrm{~mL}, 60 \mathrm{mmol})$ and 5-amino-1-pentanol $(5.10 \mathrm{~g}$, $50 \mathrm{mmol})$ was heated under reflux overnight in $\mathrm{MeOH}(100 \mathrm{~mL})$. After the reaction mixture was cooled to ambient temperature, $\mathrm{NaBH}_{4}(3.40 \mathrm{~g}, 0.09 \mathrm{~mol})$ was added portionwise to the stirring solution over a period of $0.5 \mathrm{~h}$. Stirring was maintained under ambient conditions for a further $24 \mathrm{~h}$, after which time $5 \mathrm{M} \mathrm{HCl}$ was added to the reaction mixture to neutralize superfluous $\mathrm{NaBH}_{4}$. The mixture was filtered and $\mathrm{MeOH}$ was removed with a rotaevaporator. The residue was extracted with ethyl acetate. The extract was concentrated to get a yellow residue. The residue was dissolved in $\mathrm{CH}_{2} \mathrm{Cl}_{2}$ and separated by flash column chromatography $\left(\mathrm{SiO}_{2}\right.$ : ethyl acetate/petroleum ether, $v / v 1: 5 \rightarrow$ ethyl acetate) to give $5(5.95 \mathrm{~g}, 61.7 \%)$ as a yellow oil. The proton and carbon NMR spectra of 5 are shown in Figures S8 and S9. ${ }^{1} \mathrm{H}$ NMR (400 MHz, $\left.\mathrm{CDCl}_{3}, 22{ }^{\circ} \mathrm{C}\right) \delta(\mathrm{ppm}): 7.22-7.34(5 \mathrm{H}, \mathrm{m}), 3.77(2 \mathrm{H}, \mathrm{s}), 3.56-3.60$ $(2 \mathrm{H}, \mathrm{m}), 2.63(2 \mathrm{H}, \mathrm{t}, J=7.2 \mathrm{~Hz}), 2.32(2 \mathrm{H}, \mathrm{m}), 1.50-1.58(4 \mathrm{H}, \mathrm{m}), 1.37-1.42(2 \mathrm{H}, \mathrm{m})$. ${ }^{13} \mathrm{C}$ NMR $\left(125 \mathrm{MHz}, \mathrm{CDCl}_{3}, 22{ }^{\circ} \mathrm{C}\right): \delta=139.4,128.6,128.5,127.3,62.2,53.8,49.0$, 32.5, 29.3, 23.5. HRMS: $\mathrm{m} / \mathrm{z}$ calcd for $[\mathrm{M}+\mathrm{Na}]^{+} \mathrm{C}_{12} \mathrm{H}_{19} \mathrm{NONa}, 216.1359$, found 216.1367, error 3.7 ppm. 

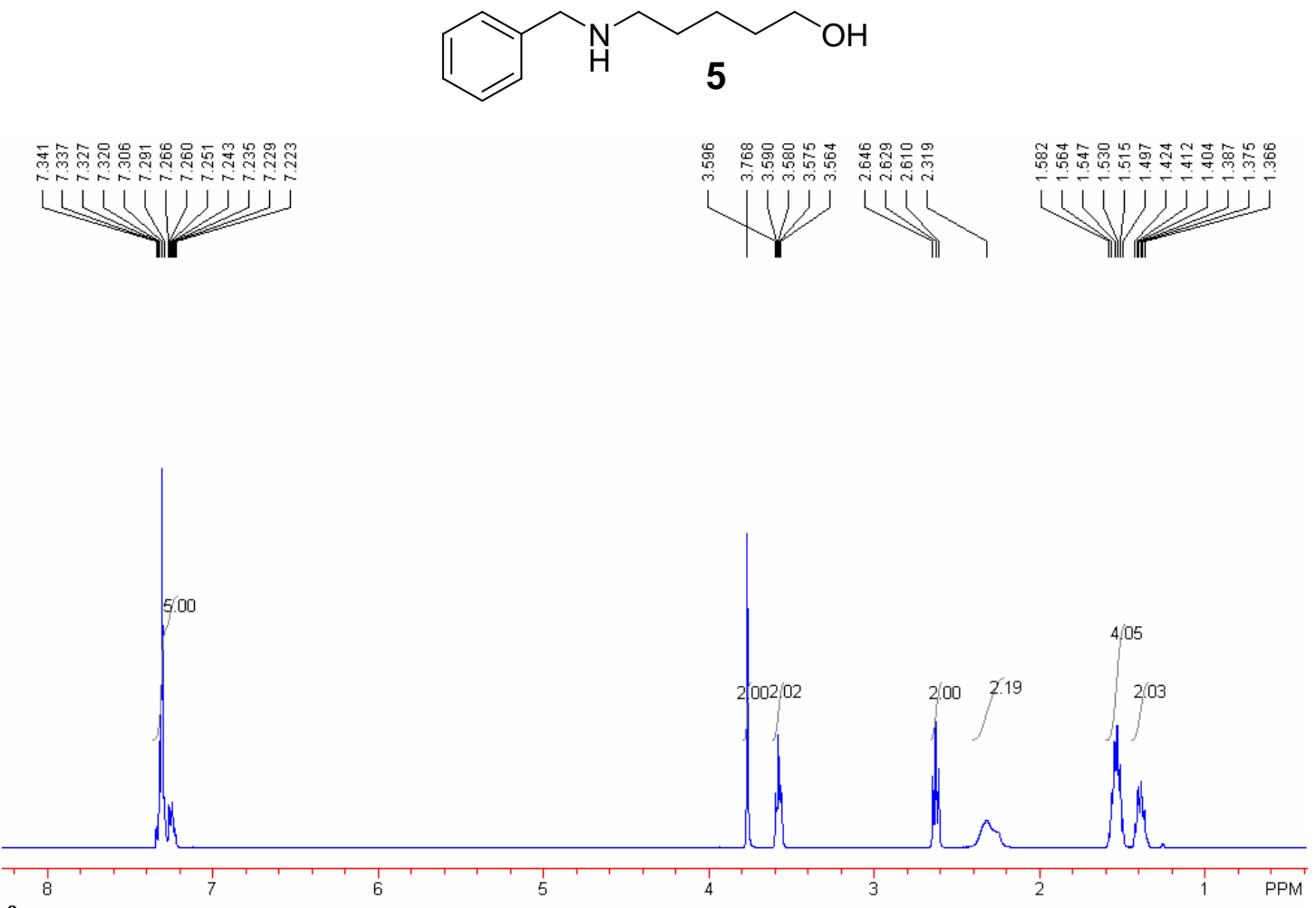

Figure S8. ${ }^{1} \mathrm{H}$ NMR spectrum $\left(400 \mathrm{MHz}, \mathrm{CDCl}_{3}, 22{ }^{\circ} \mathrm{C}\right)$ of 5 .
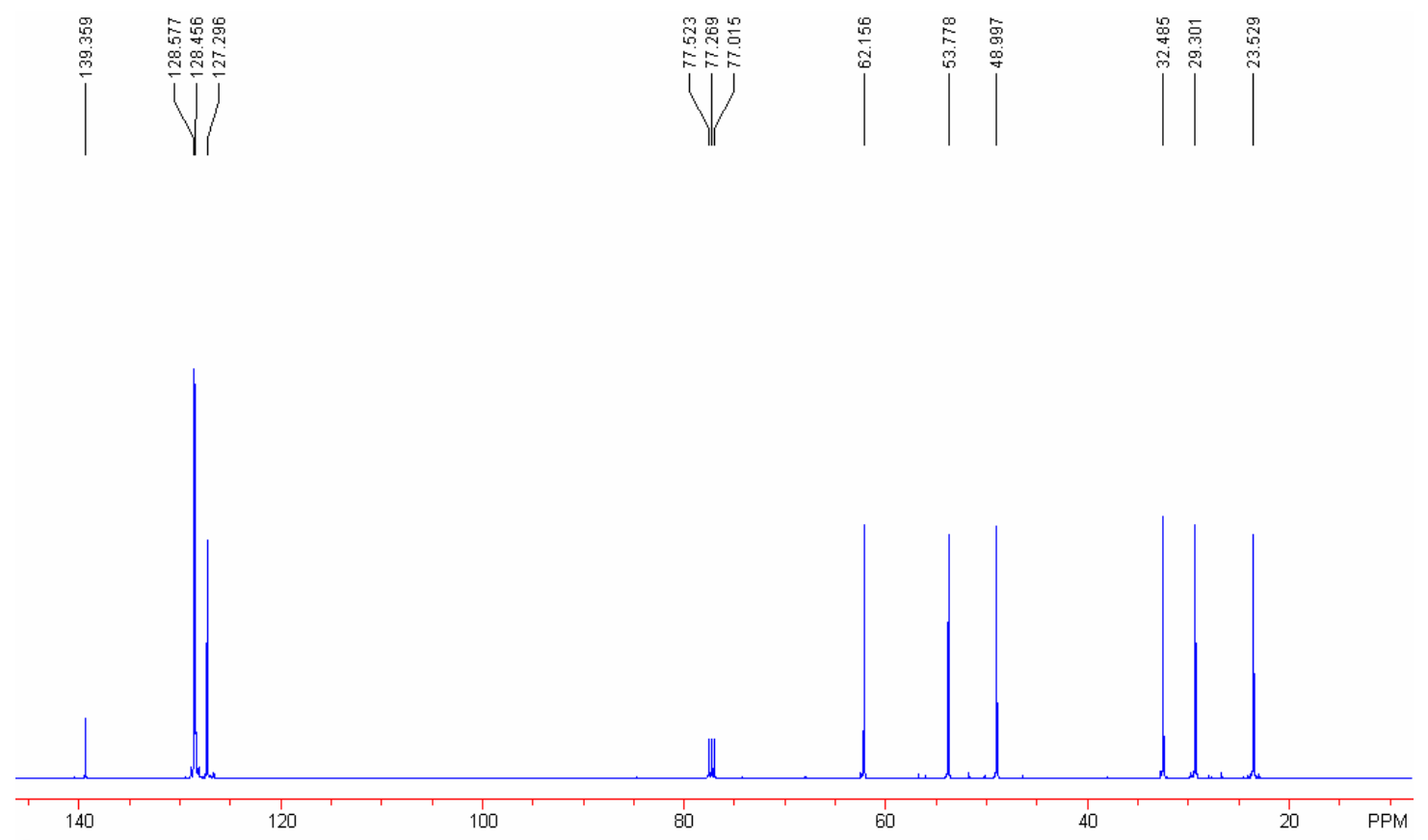

Figure S9. ${ }^{13} \mathrm{C}$ NMR spectrum $\left(125 \mathrm{MHz}, \mathrm{CDCl}_{3}, 22{ }^{\circ} \mathrm{C}\right)$ of 5 . 


\section{Synthesis of 6}

$5(2.00 \mathrm{~g}, 10.4 \mathrm{mmol})$ was dissolved in a hydrochloric acid solution until $\mathrm{pH}=6$. The solution was added to a saturated $\mathrm{NH}_{4} \mathrm{PF}_{6}$ solution to produce a precipitate, and then the mixture was concentrated. The residue was isolated by silica gel column chromatography $\left(\mathrm{SiO}_{2}\right.$ : ethyl acetate/petroleum ether, $v / v 5: 1 \rightarrow$ ethyl acetate) to afford $6(2.31 \mathrm{~g}, 66.0 \%)$ as a white solid. $\mathrm{mp} 82-84{ }^{\circ} \mathrm{C}$. The proton and carbon NMR spectra of 6 are shown in Figures S10 and S11. ${ }^{1} \mathrm{H}$ NMR $\left(400 \mathrm{MHz}, \mathrm{CD}_{3} \mathrm{COCD}_{3}, 22\right.$ $\left.{ }^{\circ} \mathrm{C}\right) \delta(\mathrm{ppm}): 7.55-7.58(2 \mathrm{H}, \mathrm{m}), 7.44-7.46(3 \mathrm{H}, \mathrm{m}), 4.48(2 \mathrm{H}, \mathrm{s}), 3.53(2 \mathrm{H}, \mathrm{t}, J=6.0$ $\mathrm{Hz}), 3.33(2 \mathrm{H}, \mathrm{t}, J=7.6 \mathrm{~Hz}), 1.85-1.89(2 \mathrm{H}, \mathrm{m}), 1.49-1.53(4 \mathrm{H}, \mathrm{m}) .{ }^{13} \mathrm{C}$ NMR $(125$ $\left.\mathrm{MHz}, \mathrm{CD}_{3} \mathrm{COCD}_{3}, 22{ }^{\circ} \mathrm{C}\right): \delta=131.4,130.1,129.6,129.1,61.1,51.9,48.4,31.8,25.8$, 22.8. HRMS: $m / z$ calcd for $\left[\mathrm{M}-\mathrm{PF}_{6}\right]^{+} \mathrm{C}_{12} \mathrm{H}_{20} \mathrm{NO}, 194.1539$, found 194.1537, error $1.0 \mathrm{ppm}$.
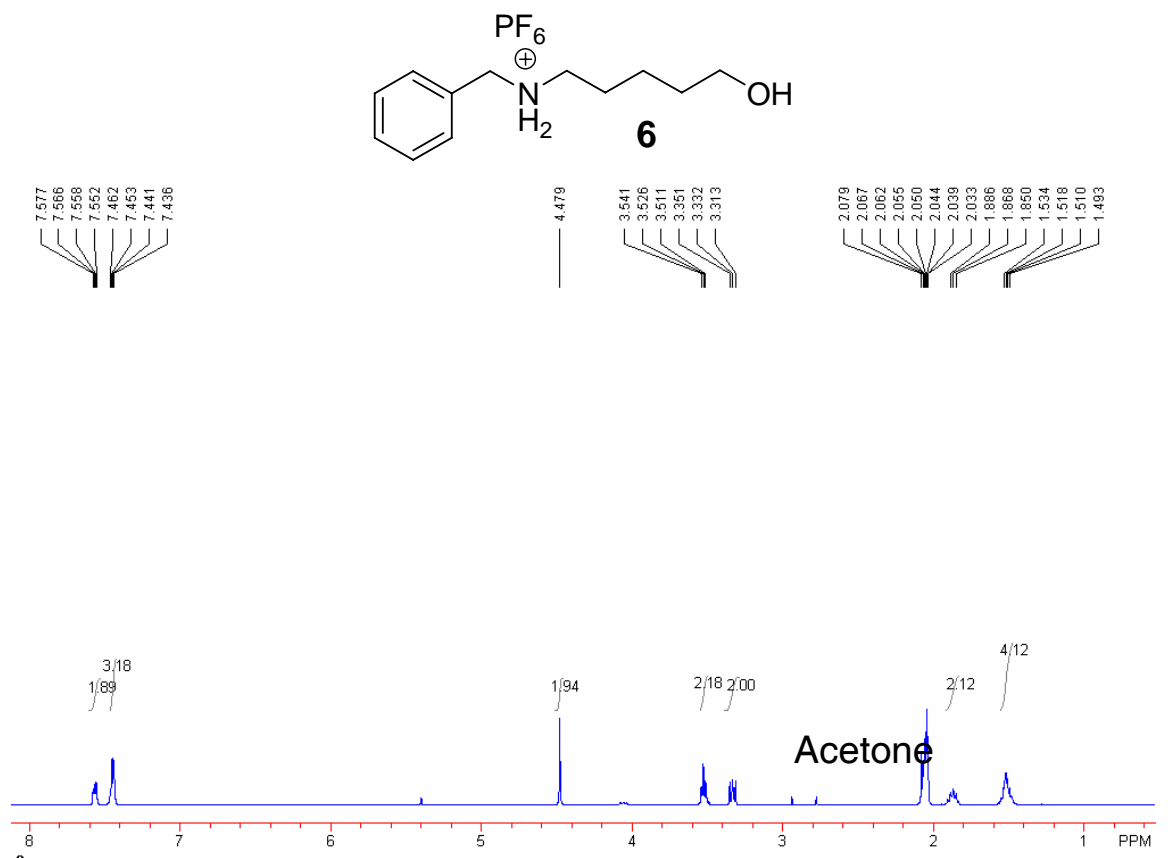

Figure S10. ${ }^{1} \mathrm{H}$ NMR spectrum $\left(400 \mathrm{MHz}, \mathrm{CD}_{3} \mathrm{COCD}_{3}, 22^{\circ} \mathrm{C}\right)$ of 6.

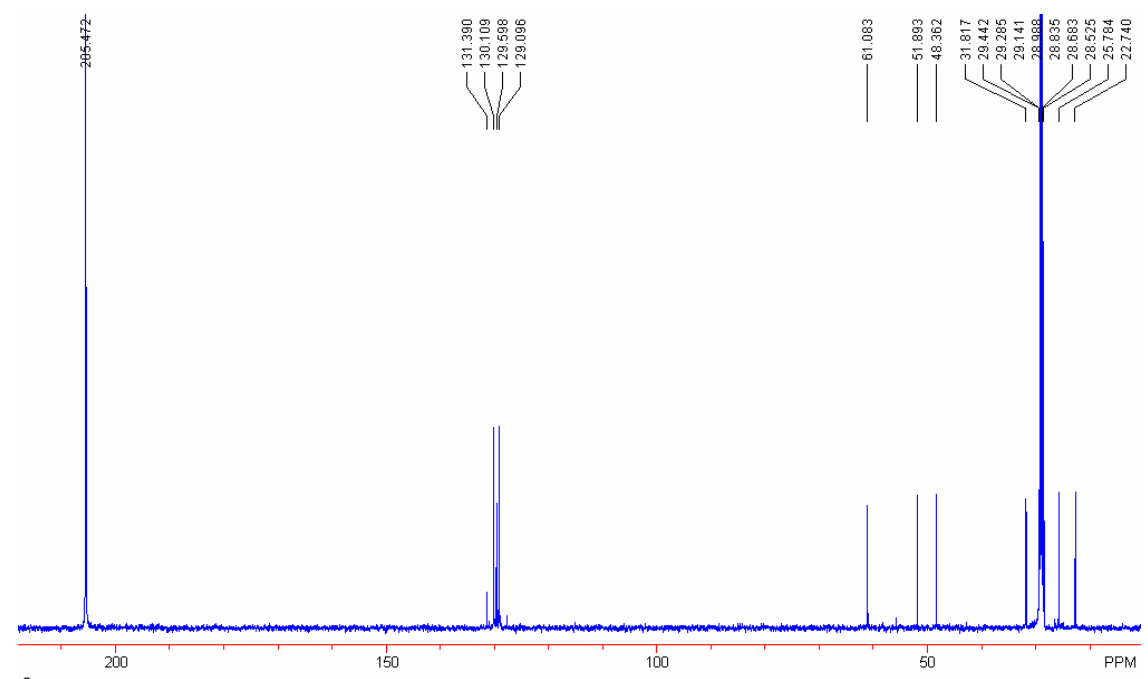

Figure S11. ${ }^{13} \mathrm{C}$ NMR spectrum $\left(125 \mathrm{MHz}, \mathrm{CD}_{3} \mathrm{COCD}_{3}, 22{ }^{\circ} \mathrm{C}\right)$ of $\mathbf{6}$. 


\section{Synthesis of dumbbell-shaped component 7}

To a solution of $6(500 \mathrm{mg}, 1.47 \mathrm{mmol})$ in acetone $(3.00 \mathrm{~mL})$ was added benzoic anhydride $(500 \mathrm{mg}, 2.20 \mathrm{mmol})$ followed by trimethylphosphine $(10.4 \mu \mathrm{L}, 0.150$ mmol). The reaction mixture was stirred for $12 \mathrm{~h}$. After evaporation, the residue was subjected to preparative silica gel column chromatography $\left(\mathrm{SiO}_{2}: \mathrm{CH}_{2} \mathrm{Cl}_{2}\right)$ to afford 7 (280 mg, 43.0\%) as a white solid. $\mathrm{mp} 183-185^{\circ} \mathrm{C}$. The proton and carbon NMR spectra of 7 are shown in Figures S12 and S13. ${ }^{1} \mathrm{H}$ NMR $\left(400 \mathrm{MHz}, \mathrm{CDCl}_{3}, 22{ }^{\circ} \mathrm{C}\right) \delta$ (ppm): $8.01(2 \mathrm{H}, \mathrm{d}, J=7.2 \mathrm{~Hz}), 7.55(1 \mathrm{H}, \mathrm{t}, J=7.4 \mathrm{~Hz}), 7.43(2 \mathrm{H}, \mathrm{t}, J=7.6 \mathrm{~Hz})$, $7.33-7.37(5 \mathrm{H}, \mathrm{m}), 4.29(2 \mathrm{H}, \mathrm{t}, J=6.4 \mathrm{~Hz}), 4.00(2 \mathrm{H}, \mathrm{s}), 2.82(2 \mathrm{H}, \mathrm{t}, J=7.6 \mathrm{~Hz})$, $1.67-1.78(4 \mathrm{H}, \mathrm{m}), 1.43-1.49(2 \mathrm{H}, \mathrm{m}) .{ }^{13} \mathrm{C} \mathrm{NMR}\left(100 \mathrm{MHz}, \mathrm{CD}_{3} \mathrm{COCD}_{3}, 22{ }^{\circ} \mathrm{C}\right): \delta=$ 165.8, 132.9, 132.1, 130.3, 129.7, 129.1, 128.8, 128.4, 64.1, 51.8, 48.0, 27.9, 25.8, 22.7. HRESIMS: $\mathrm{m} / \mathrm{z}$ calcd for $\left[\mathrm{M}-\mathrm{PF}_{6}\right]^{+} \mathrm{C}_{19} \mathrm{H}_{24} \mathrm{NO}_{2}, 298.1802$, found 298.1809, error $2.3 \mathrm{ppm}$.
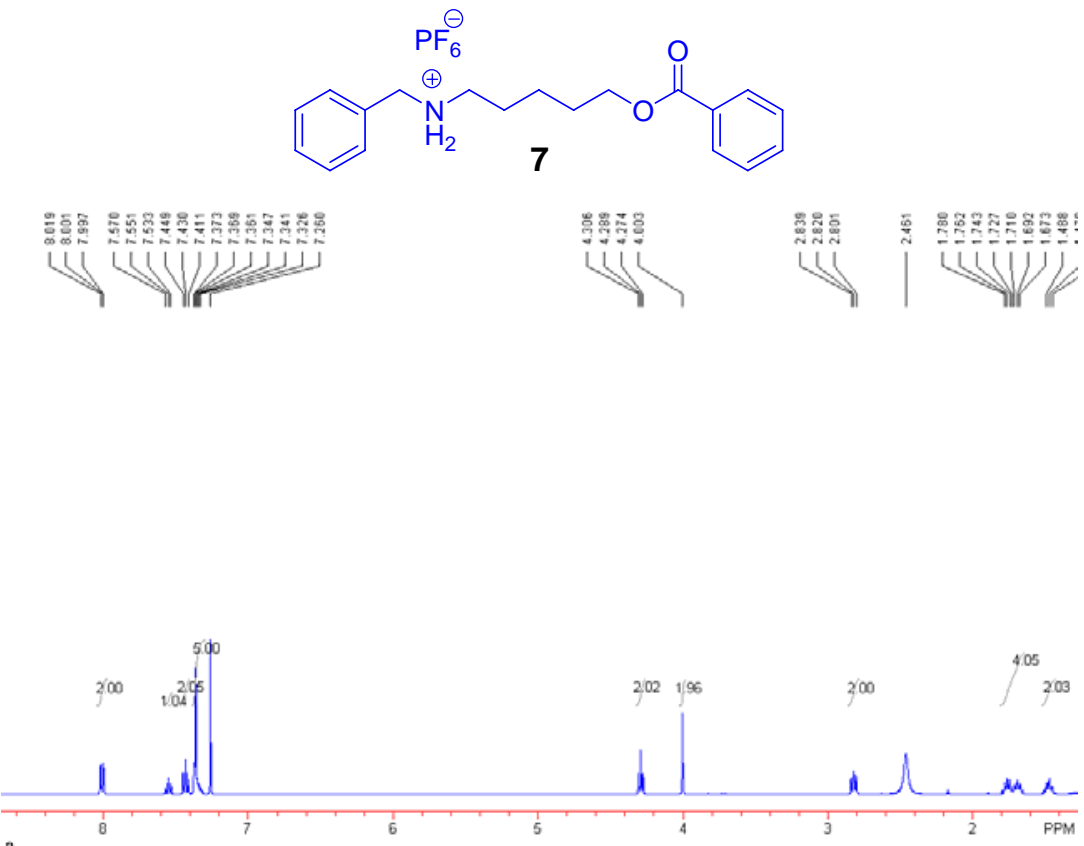

Figure S12. ${ }^{1} \mathrm{H}$ NMR spectrum $\left(400 \mathrm{MHz}, \mathrm{CDCl}_{3}, 22{ }^{\circ} \mathrm{C}\right)$ of 7 .

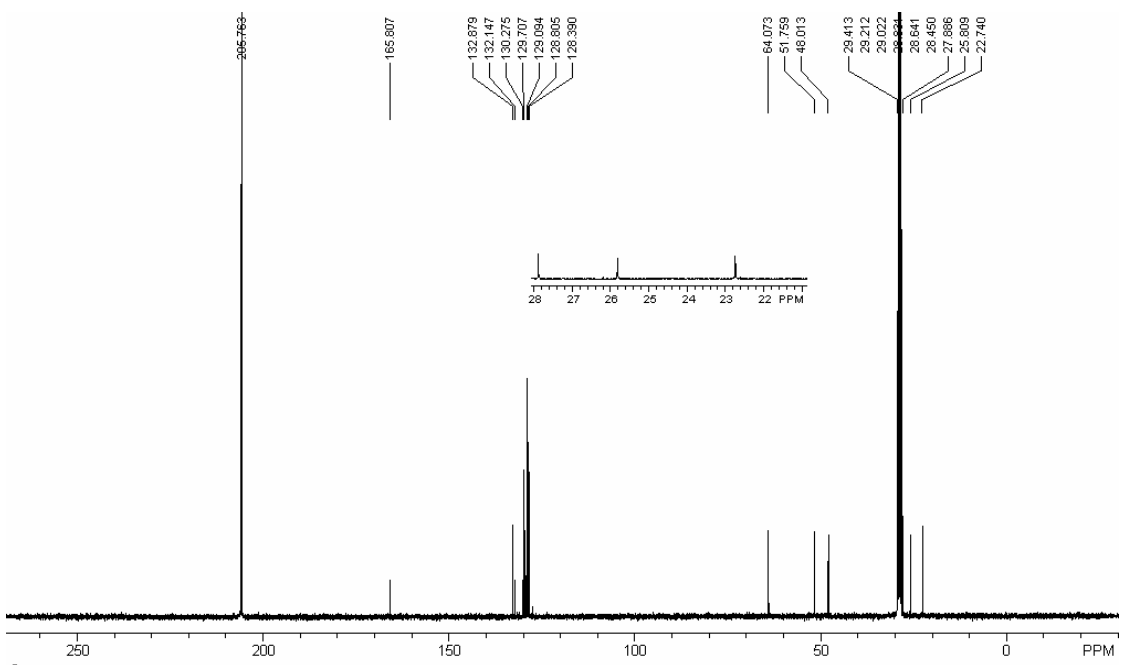

Figure S13. ${ }^{13} \mathrm{C}$ NMR spectrum $\left(100 \mathrm{MHz}, \mathrm{CD}_{3} \mathrm{COCD}_{3}, 22{ }^{\circ} \mathrm{C}\right)$ of 7 . 


\section{Synthesis of rotaxane 8}

To a solution of $6(500 \mathrm{mg}, 1.47 \mathrm{mmol})$ and benzo-21-crown-7 (530 mg, 1.47 mmol) in dichloromethane $(5.00 \mathrm{~mL})$ was added benzoic anhydride $(500 \mathrm{mg}, 2.20$ mmol) followed by trimethylphosphine $(10.4 \mu \mathrm{L}, 0.150 \mathrm{mmol})$. The reaction mixture was stirred for $12 \mathrm{~h}$. After evaporation, the residue was subjected to silica gel column chromatography $\left(\mathrm{SiO}_{2}: \mathrm{CH}_{2} \mathrm{Cl}_{2}\right)$ to give rotaxane $8(0.860 \mathrm{~g}, 74 \%)$ as a white solid. $\mathrm{mp} 130-132{ }^{\circ} \mathrm{C}$. The proton and carbon NMR spectra of the rotaxane 8 are shown in Figures S14 and S15. ${ }^{1} \mathrm{H}$ NMR (400 MHz, $\left.\mathrm{CDCl}_{3}, 22{ }^{\circ} \mathrm{C}\right) \delta(\mathrm{ppm}): 8.01-8.03(2 \mathrm{H}, \mathrm{m})$, $7.56(3 \mathrm{H}, \mathrm{t}, J=7.4 \mathrm{~Hz}), 7.43(2 \mathrm{H}, \mathrm{t}, J=7.8 \mathrm{~Hz}), 7.28-7.36(5 \mathrm{H}, \mathrm{m}), 6.86-6.93(4 \mathrm{H}$, $\mathrm{m}), 4.16-4.38(8 \mathrm{H}, \mathrm{m}), 3.87-3.91(2 \mathrm{H}, \mathrm{m}), 3.68-3.77(4 \mathrm{H}, \mathrm{m}), 3.74-3.64(16 \mathrm{H}, \mathrm{m})$, $1.71-1.78(2 \mathrm{H}, \mathrm{m}), 1.57-1.63(2 \mathrm{H}, \mathrm{m}), 1.41-1.49(2 \mathrm{H}, \mathrm{m}) .{ }^{13} \mathrm{C}$ NMR $(125 \mathrm{MHz}$, $\left.\mathrm{CDCl}_{3}, 22{ }^{\circ} \mathrm{C}\right): \delta=150.0,133.3,132.7,130.4,129.7,129.1,128.7,121.9,112.0,71.6$, $71.3,71.1,70.8,70.0,68.4,64.4,51.3,47.3,28.4,26.4,23.8$. ESI-MS is shown in Figure S16: $\mathrm{m} / \mathrm{z} 654.4(100 \%)\left[\mathrm{M}-\mathrm{PF}_{6}\right]^{+}$.
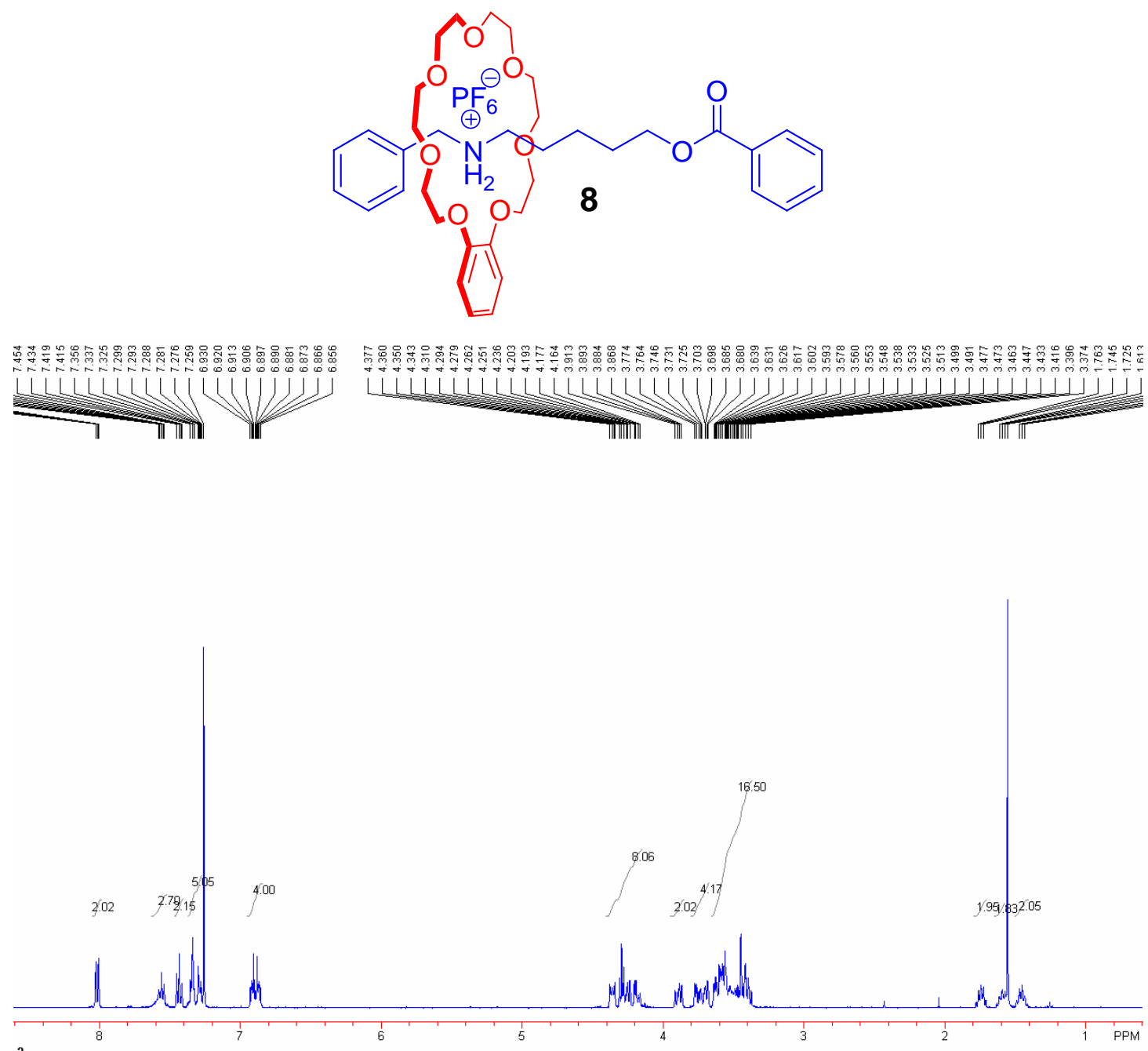

Figure S14. ${ }^{1} \mathrm{H}$ NMR spectrum $\left(400 \mathrm{MHz}, \mathrm{CDCl}_{3}, 22^{\circ} \mathrm{C}\right)$ of rotaxane 8 . 

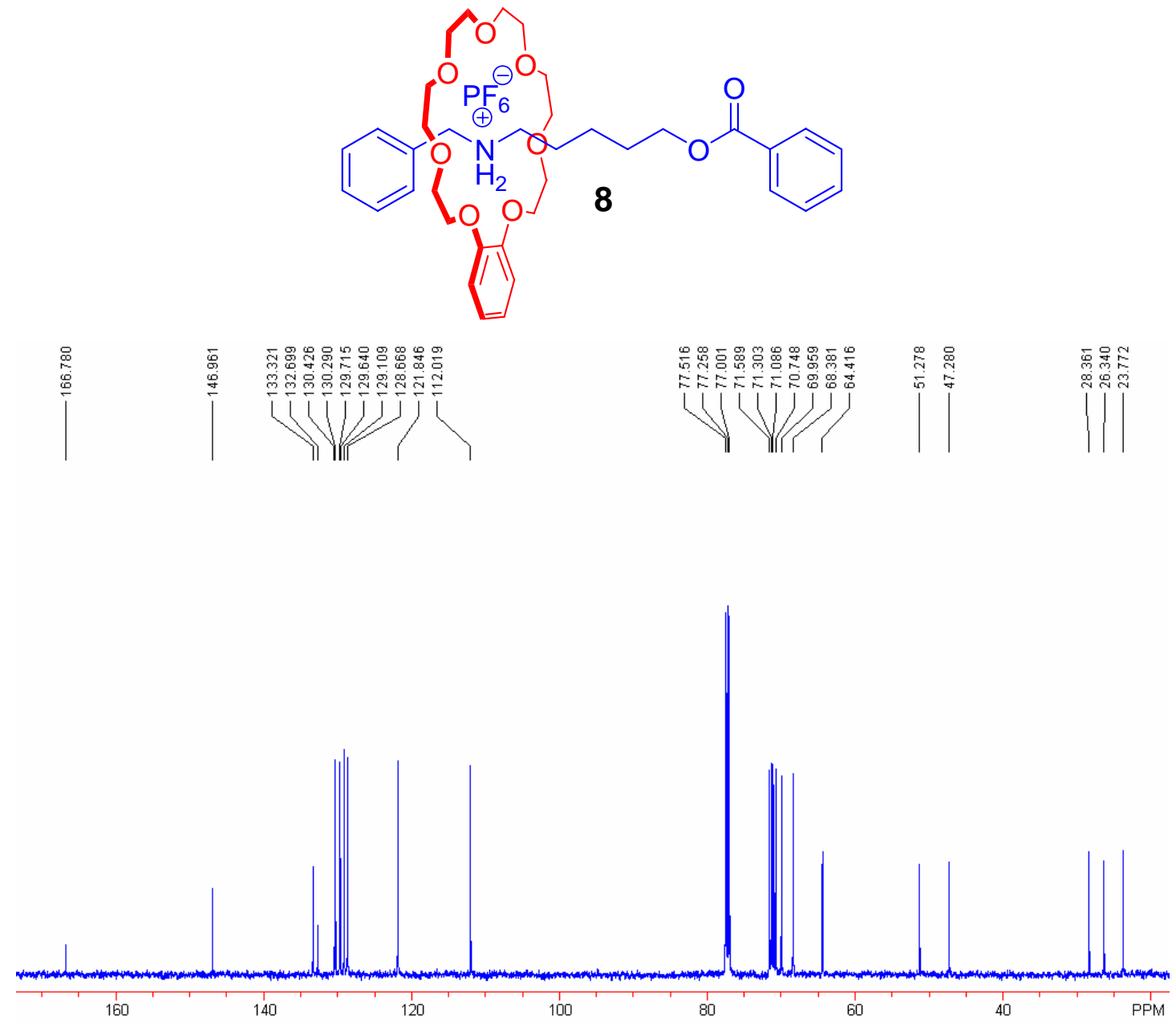

Figure S15. ${ }^{13} \mathrm{C}$ NMR spectrum $\left(125 \mathrm{MHz}, \mathrm{CDCl}_{3}, 22{ }^{\circ} \mathrm{C}\right)$ of rotaxane 8 . 


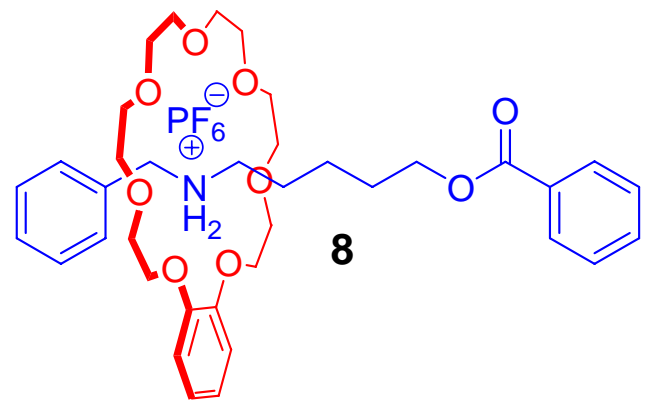

Display Report $\begin{array}{ll}\text { Analysis Info } & \\ \text { Analysis Name } & \text { 07071722.d } \\ \text { Method } & \text { Copy of 070602.MS }\end{array}$

Sample Name ZCJ - 39 Acquisition Date $\quad$ 07/17/07 15:46:37

Acquisition Parameter Ion Source Type ESI

Mass Range Mode Std/Norm

Capillary Exi! 280.0 Vol Scan Begin Averages
Positive
$103 \mathrm{~m} / \mathrm{z}$
$40.0 \mathrm{Volt}$

8 Spectra

Alternating lon Polarity Scan End
Trap Drive

Trap Drive
Auto MS/MS Operator Administrator

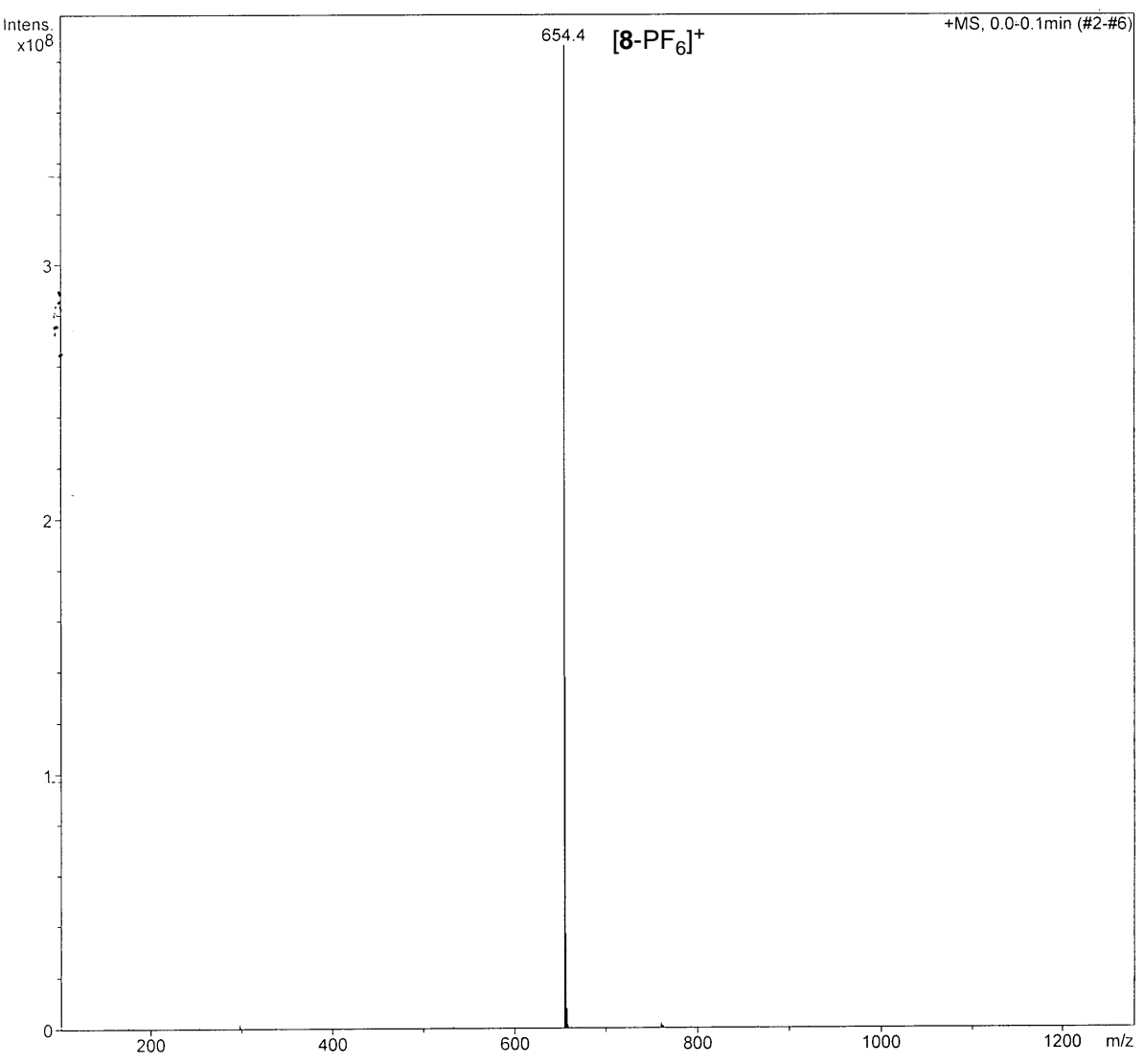

Bruker Daltonics DataAnalysis 3.1

printed:

$07 / 17 / 07$

$16: 02: \overline{8}$

Pagë|1 of 1

Figure S16. Electrospray ionization mass spectrum of rotaxane 8. m/z $654.4(100 \%)$ $\left[\mathrm{M}-\mathrm{PF}_{6}\right]^{+}$. 
9. Partial proton NMR spectra of dumbbell-shaped component 7 , rotaxane 8 and rotaxane 8 neutralized by $\mathrm{Et}_{3} \mathrm{~N}$.
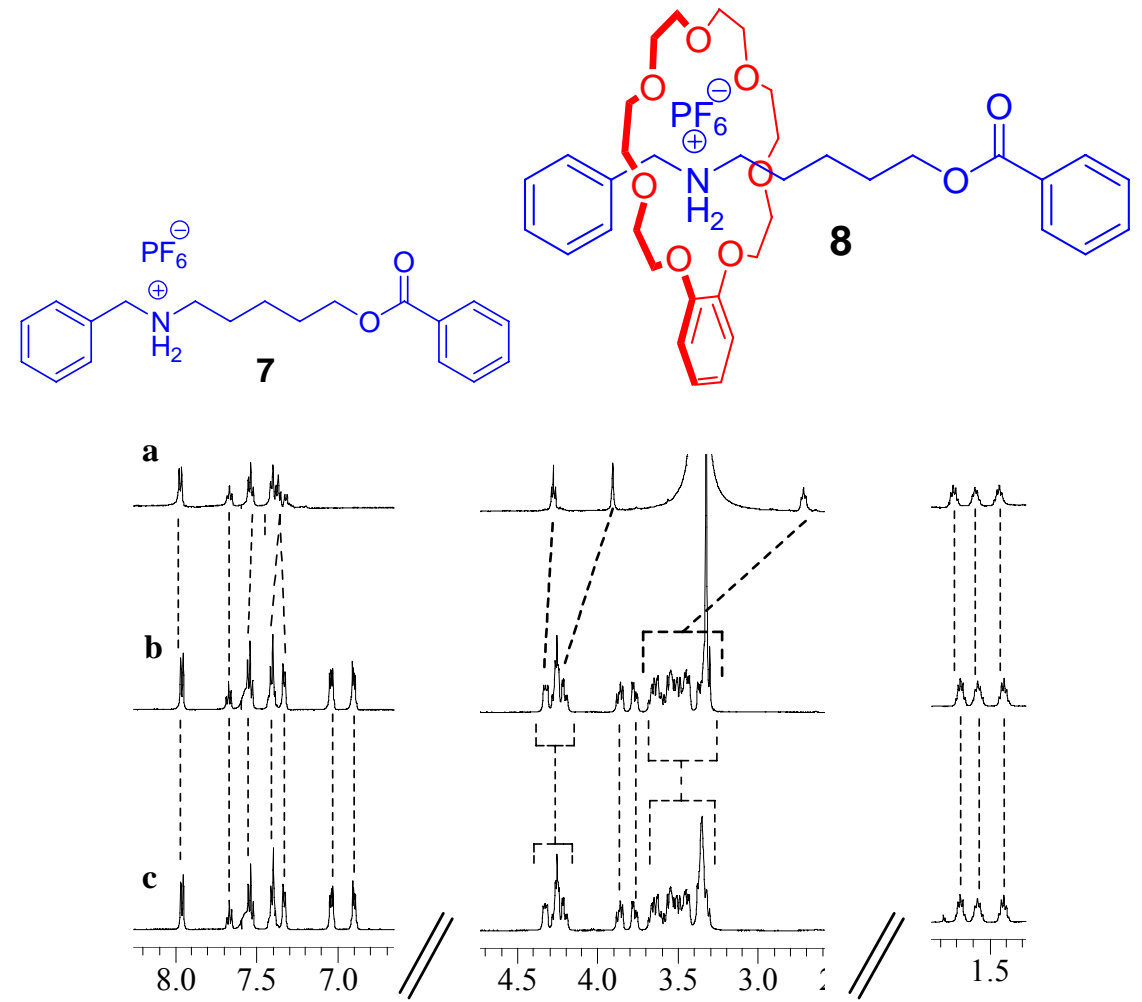

Figure S17. Partial proton NMR spectra $\left(500 \mathrm{MHz}\right.$, DMSO- $\left.d_{6}, 22{ }^{\circ} \mathrm{C}\right)$ of (a) dumbbell-shaped component 7, (b) rotaxane 8. (c) rotaxane 8 neutralized by $\mathrm{Et}_{3} \mathrm{~N}$. No chemical shift changes were observed after rotaxane $\mathbf{8}$ was neutralized.

\section{X-ray analysis data of rotaxane 8}

Crystallographic data: block, colorless, $0.54 \times 0.42 \times 0.36 \mathrm{~mm}^{3}, \mathrm{C}_{37} \mathrm{H}_{52} \mathrm{NO}_{9} \mathrm{~F}_{6} \mathrm{P}, \mathrm{FW}$ 799.77, triclinic, space group $P^{\overline{1}}, a=11.775(2), b=14.080(3), c=14.801(3) \AA, \alpha=$ $117.56(3)^{\circ}, \beta=109.34(3)^{\circ}, \gamma=92.84(3)^{\circ}, V=1991.5(11) \AA^{3}, Z=2, D_{\mathrm{c}}=1.334 \mathrm{~g} \mathrm{~cm}^{-3}$, $T=295(2) \mathrm{K}, \mu=0.150 \mathrm{~mm}^{-1}, 12156$ measured reflections, 5185 independent reflections, 487 parameters, 0 restraints, $F(000)=844, R_{1}=0.1048, w R_{2}=0.2866$ (all data), $R_{1}=0.0922, w R_{2}=0.2734[I>2 \sigma(I)]$, max. residual density $0.917 \mathrm{e} \bullet \AA^{-3}$, and goodness-of-fit $\left(F^{2}\right)=1.095$. 
11. ${ }^{1} \mathrm{H}$ NMR Spectrum of an equimolar acetone solution of B18C6 and 1

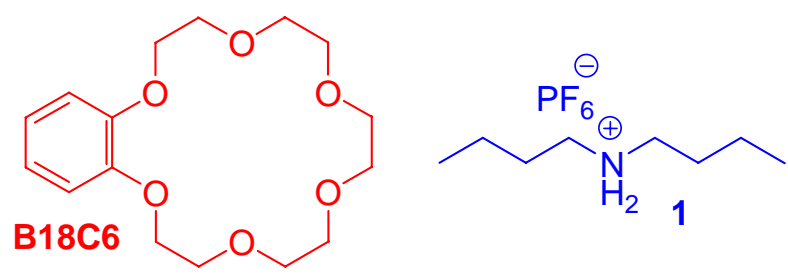

a

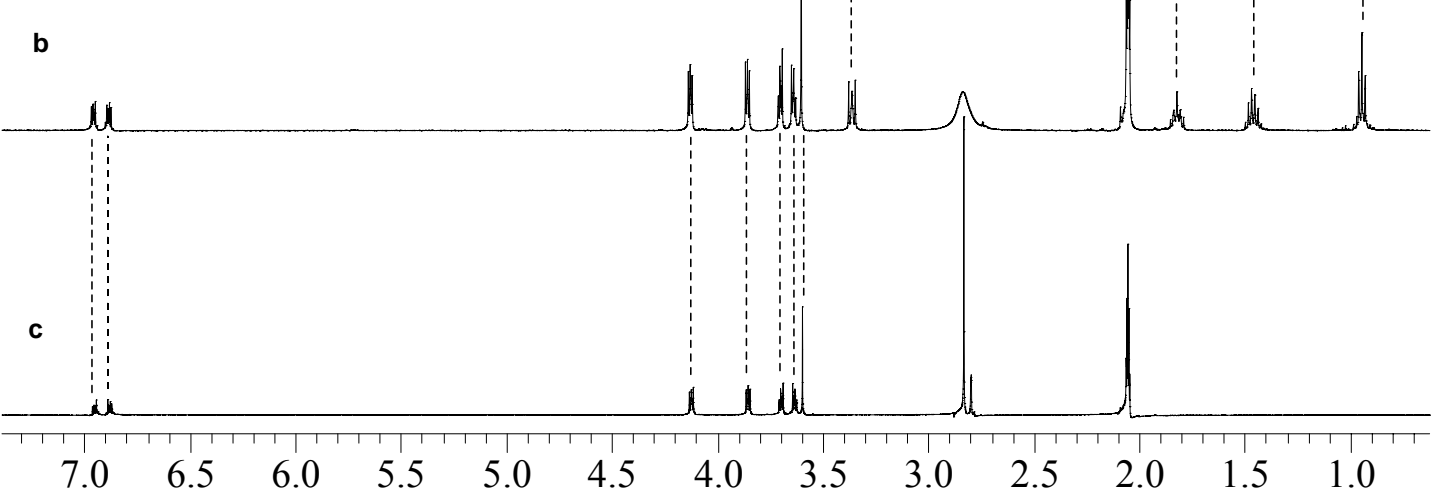

Figure S18. ${ }^{1} \mathrm{H}$ NMR spectra $\left(500 \mathrm{MHz}, \mathrm{CD}_{3} \mathrm{COCD}_{3}, 22{ }^{\circ} \mathrm{C}\right)$ of (a) $4.00 \mathrm{mM} \mathrm{1}$, (b) $4.00 \mathrm{mM}$ B18C6 and 1, (c) $4.00 \mathrm{mM}$ B18C6. The spectrum demonstrates that B18C6 can not complex $\mathbf{1}$ because neither chemical shift changes nor signal doubling occurred upon mixing B18C6 and $\mathbf{1 .}$ 


\section{2. ${ }^{1} \mathrm{H}$ NMR Spectrum of an equimolar acetone solution of B21C7 and 4}

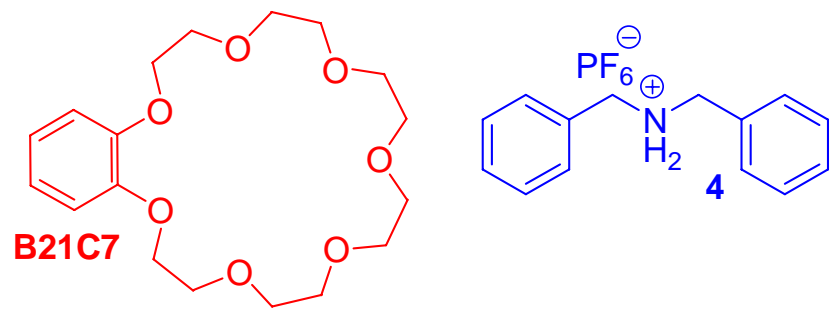

a

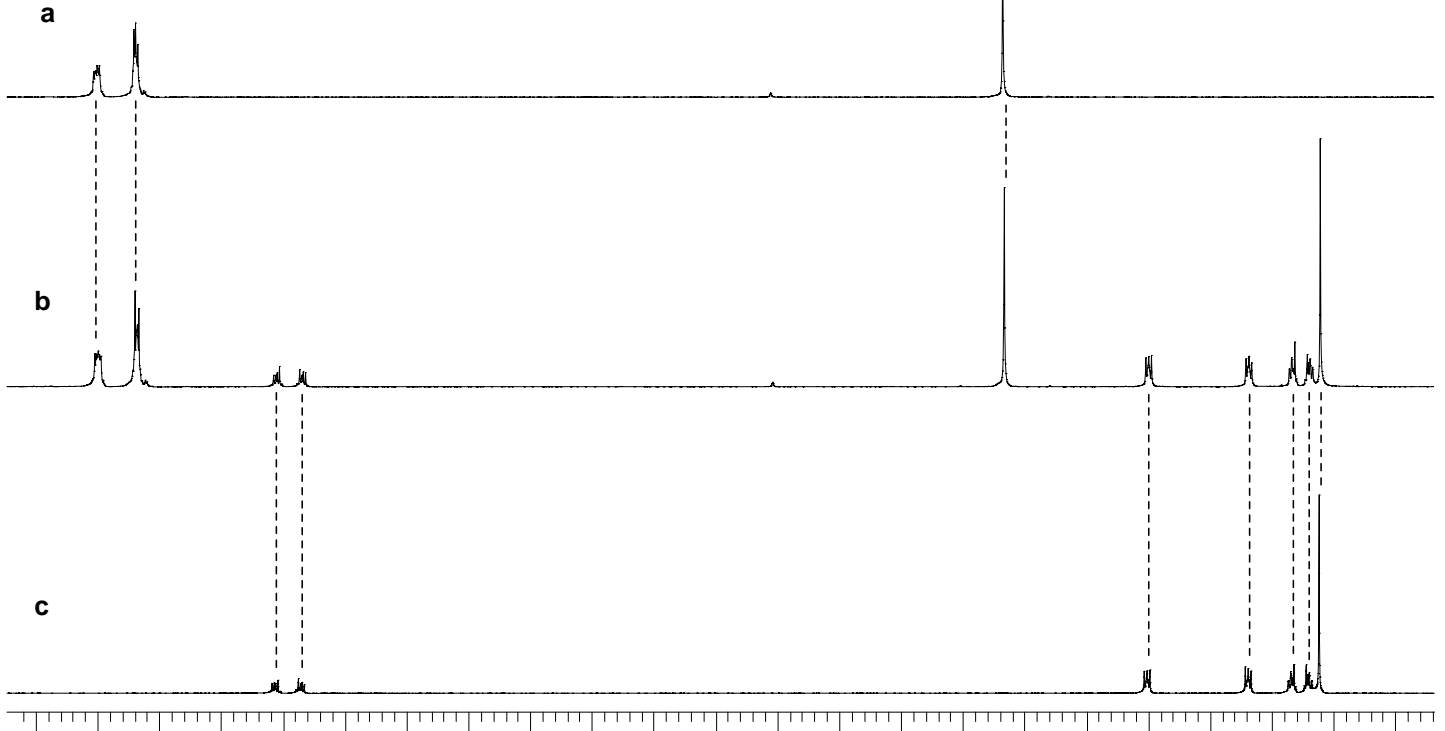

7.8 7.6 7.4 7.2 7.0 6.8 6.6 6.4 6.2 6.0 5.8 5.6 5.4 5.2 5.0 4.8 4.6 4.4 4.2 4.0 3.8 3.6 3.4

Figure S19. ${ }^{1} \mathrm{H}$ NMR spectra $\left(500 \mathrm{MHz}, \mathrm{CD}_{3} \mathrm{COCD}_{3}, 22{ }^{\circ} \mathrm{C}\right.$ ) of (a) $4.00 \mathrm{mM} \mathrm{4}$, (b) $4.00 \mathrm{mM}$ B21C7 and 4, (c) $4.00 \mathrm{mM}$ B21C7. The spectra demonstrates that B21C7 can not complex 4 because neither chemical shift changes nor signal doubling occurred upon mixing B21C7 and 4. 


\section{3. ${ }^{1} \mathrm{H}$ NMR Spectrum and ESI-MS of an equimolar acetone solution of B21C7 and}

1
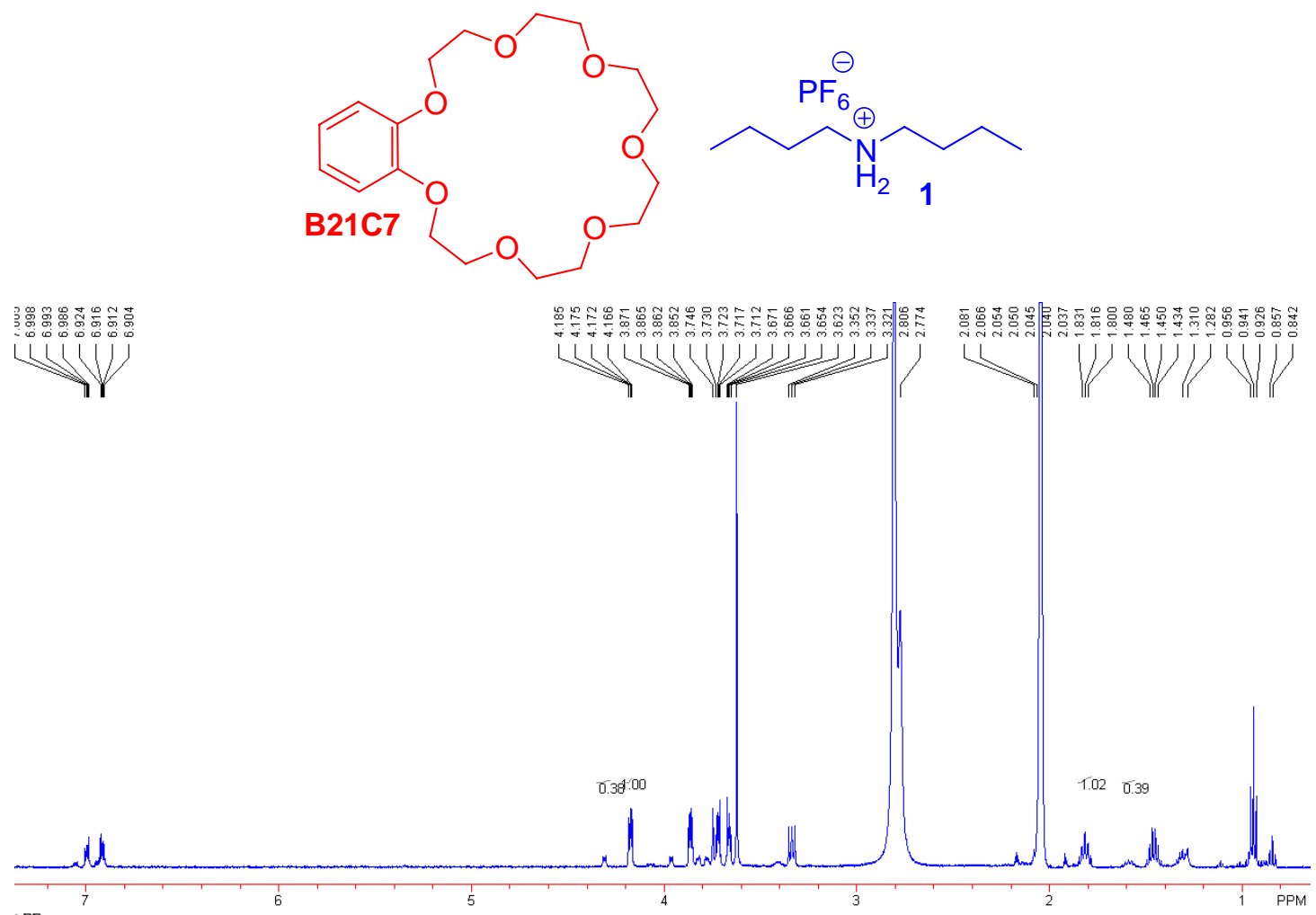

Figure S20. ${ }^{1} \mathrm{H}$ NMR spectrum $\left(500 \mathrm{MHz}, \mathrm{CD}_{3} \mathrm{COCD}_{3}, 22{ }^{\circ} \mathrm{C}\right)$ of $1.00 \mathrm{mM} \mathbf{B 2 1 C 7}$ and 1. The association constant $K_{\mathrm{a}, \mathbf{B} 21 \mathbf{C 7} \mathbf{1}}$ value calculated from integrations of complexed and uncomplexed peaks of $\mathrm{H}_{3}$ of B21C7 is $\left[(0.38 / 1.38) \times 1.00 \times 10^{-3}\right] /[(1-0.38 / 1.38) \times 1.00 \times$ $\left.10^{-3}\right]^{2}=523 \mathrm{M}^{-1}$. The association constant $K_{\mathrm{a}, \mathbf{B} 21 \mathrm{C7} \cdot \mathbf{1}}$ value calculated from integrations of complexed and uncomplexed peaks of $\beta$-methylene protons of 1 is $[(0.39 / 1.41) \times 1.00 \times$ $\left.10^{-3}\right] /\left[(1-0.39 / 1.41) \times 1.00 \times 10^{-3}\right]^{2}=530 \mathrm{M}^{-1}$. Therefore, $K_{\mathrm{a}, \mathbf{B} 21 \mathbf{C 7} \mathbf{\bullet 1}}=(523+530) / 2=527( \pm$ 4) $\mathrm{M}^{-1}$. 


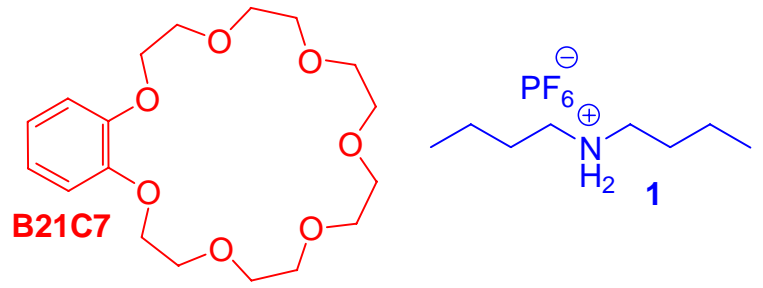

Display Report
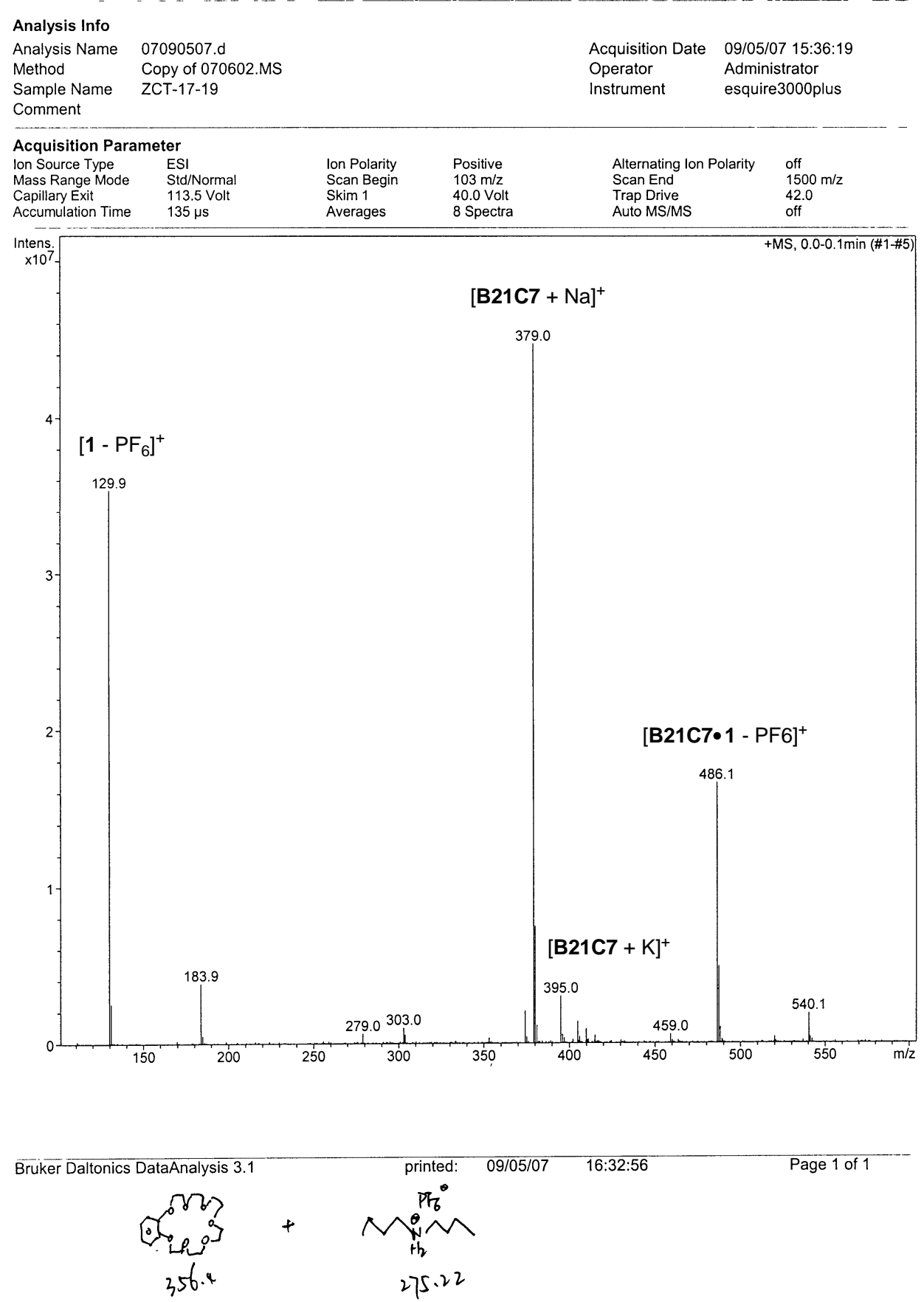

Figure S21. Electrospray ionization mass spectrum of a solution of B21C7 and 1 (1:1 molar ratio). $m / z 129.9(79 \%)\left[\mathbf{1}-\mathrm{PF}_{6}\right]^{+}, 379.0(100 \%)[\mathbf{B} 21 \mathrm{C} 7+\mathrm{Na}]^{+}, 395.0(7 \%)$ $[\mathbf{B 2 1 C 7}+\mathrm{K}]^{+}, 486.1(37 \%)\left[\mathbf{B 2 1 C 7} 1-\mathrm{PF}_{6}\right]^{+}$. 

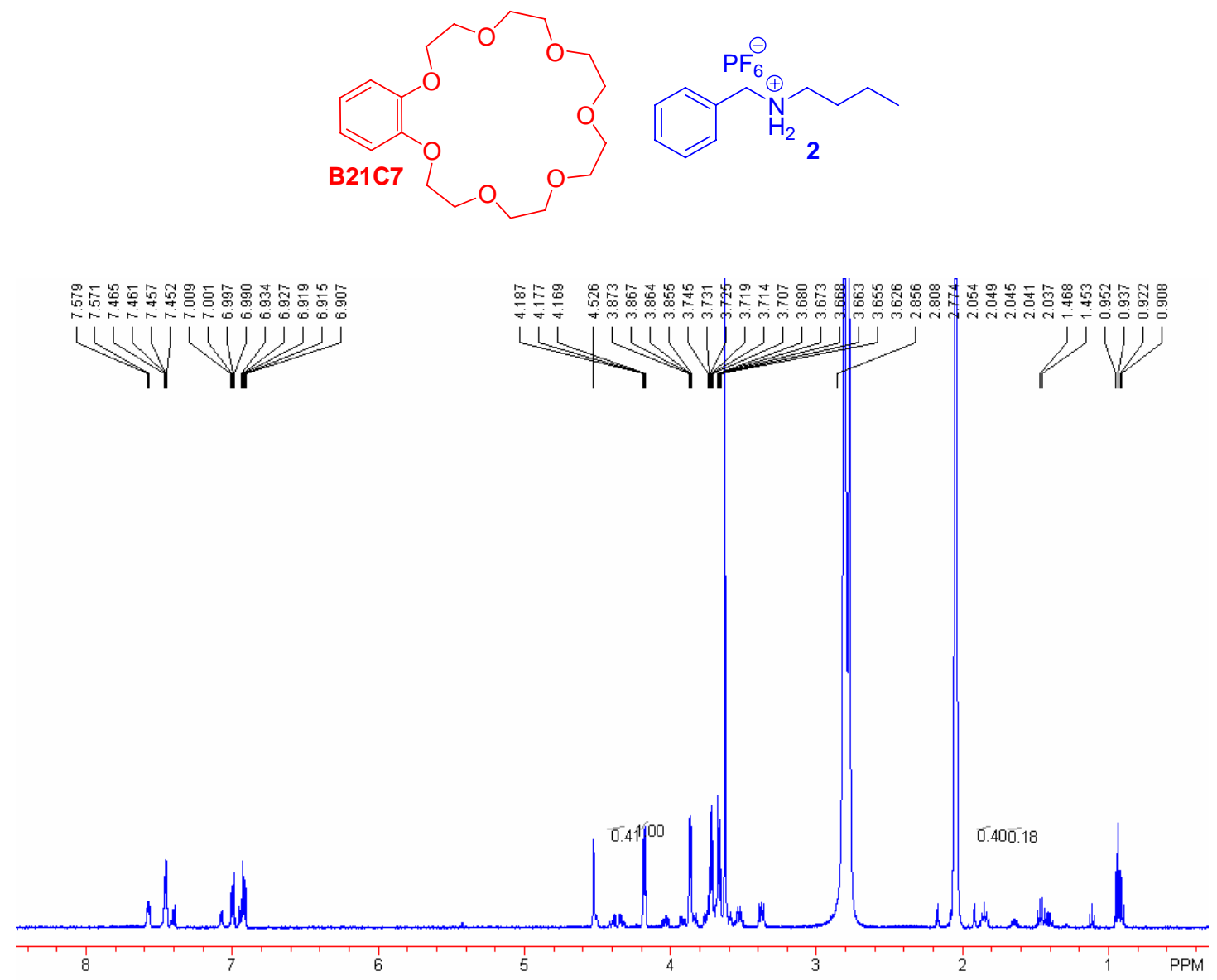

Figure S22. ${ }^{1} \mathrm{H}$ NMR spectrum $\left(500 \mathrm{MHz}, \mathrm{CD}_{3} \mathrm{COCD}_{3}, 22{ }^{\circ} \mathrm{C}\right)$ of $1.00 \mathrm{mM} \mathbf{B 2 1 C 7}$ and 2. The association constant $K_{\mathrm{a}, \mathbf{B} 21 \mathbf{C 7} \cdot 2}$ value calculated from integrations of complexed and uncomplexed peaks of $\mathrm{H}_{3}$ of B21C7 is $\left[(0.41 / 1.41) \times 1.00 \times 10^{-3}\right] /[(1-0.41 / 1.41) \times 1.00 \times$

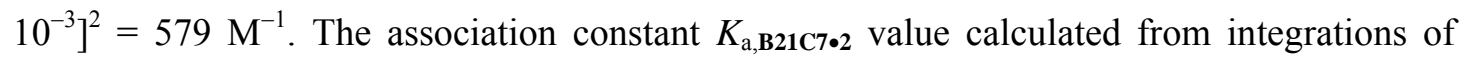
complexed and uncomplexed peaks of $\beta$-methylene protons of 2 is $[(0.18 / 0.58) \times 1.00 \times$ $\left.10^{-3}\right] /\left[(1-0.18 / 0.58) \times 1.00 \times 10^{-3}\right]^{2}=651 \mathrm{M}^{-1}$. Therefore, $K_{\mathrm{a}, \mathbf{B 2 1 C 7} \bullet 2}=(579+651) / 2=615$ $( \pm 36) \mathrm{M}^{-1}$. 


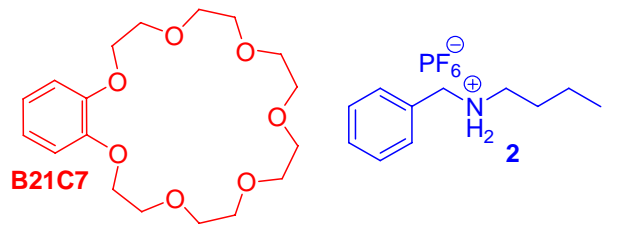

Display Report
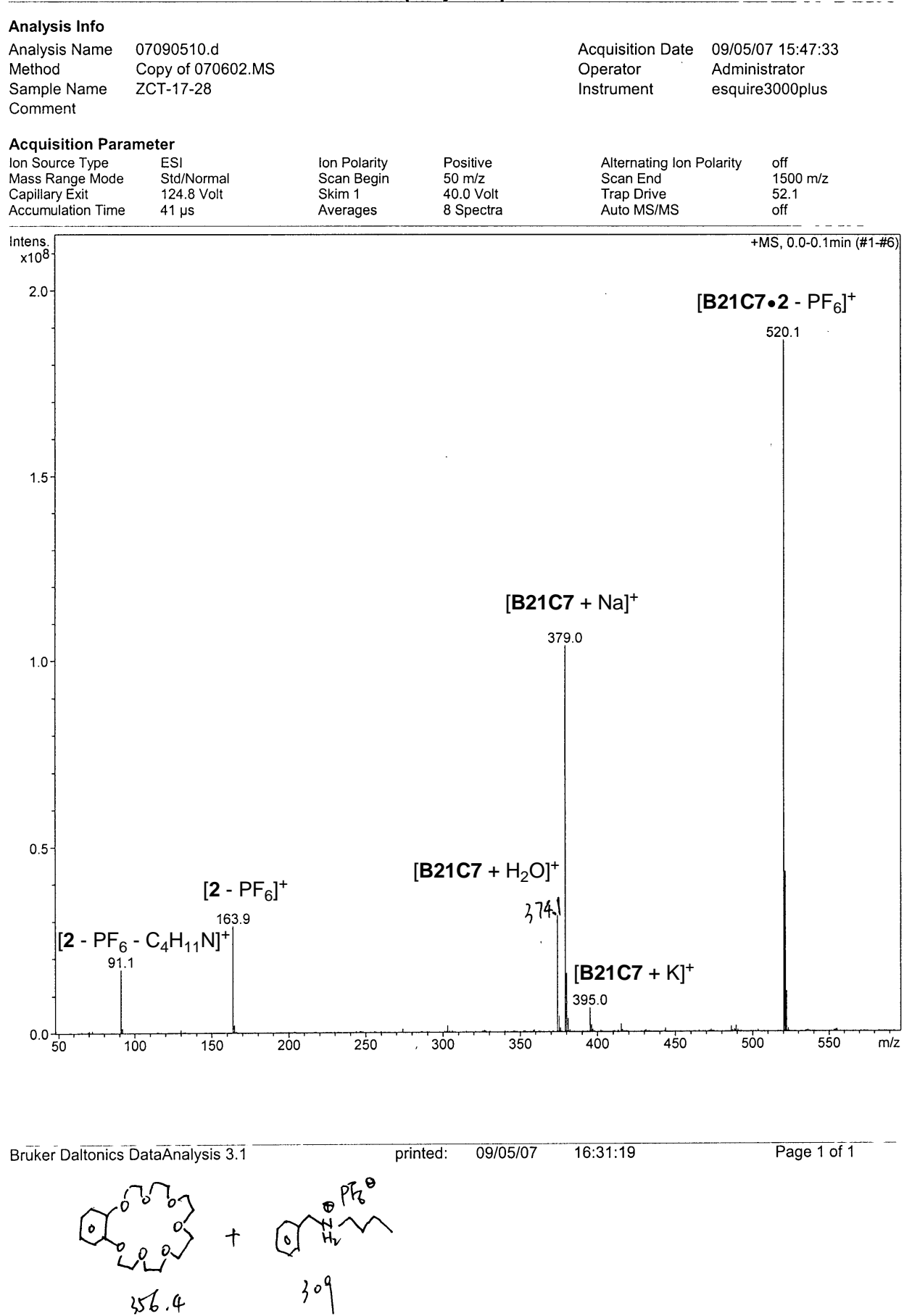

Figure S23. Electrospray ionization mass spectrum of a solution of B21C7 and 2 (1:1 molar ratio). $m / z 91.1(20 \%)\left[2-\mathrm{PF}_{6}-\mathrm{C}_{4} \mathrm{H}_{11} \mathrm{~N}\right]^{+}, 163.9(15 \%)\left[2-\mathrm{PF}_{6}\right]^{+}, 374.1$ $(17 \%)\left[\mathbf{B} 21 \mathrm{C} 7+\mathrm{H}_{2} \mathrm{O}\right]^{+}, 395.0(56 \%)[\mathbf{B 2 1 C 7}+\mathrm{Na}]^{+}, 395.0(3 \%)[\mathbf{B 2 1 C} 7+\mathrm{K}]^{+}$, $520.1(100 \%)\left[\mathbf{B} 21 \mathbf{C} 7 \cdot 2-\mathrm{PF}_{6}\right]^{+}$. 


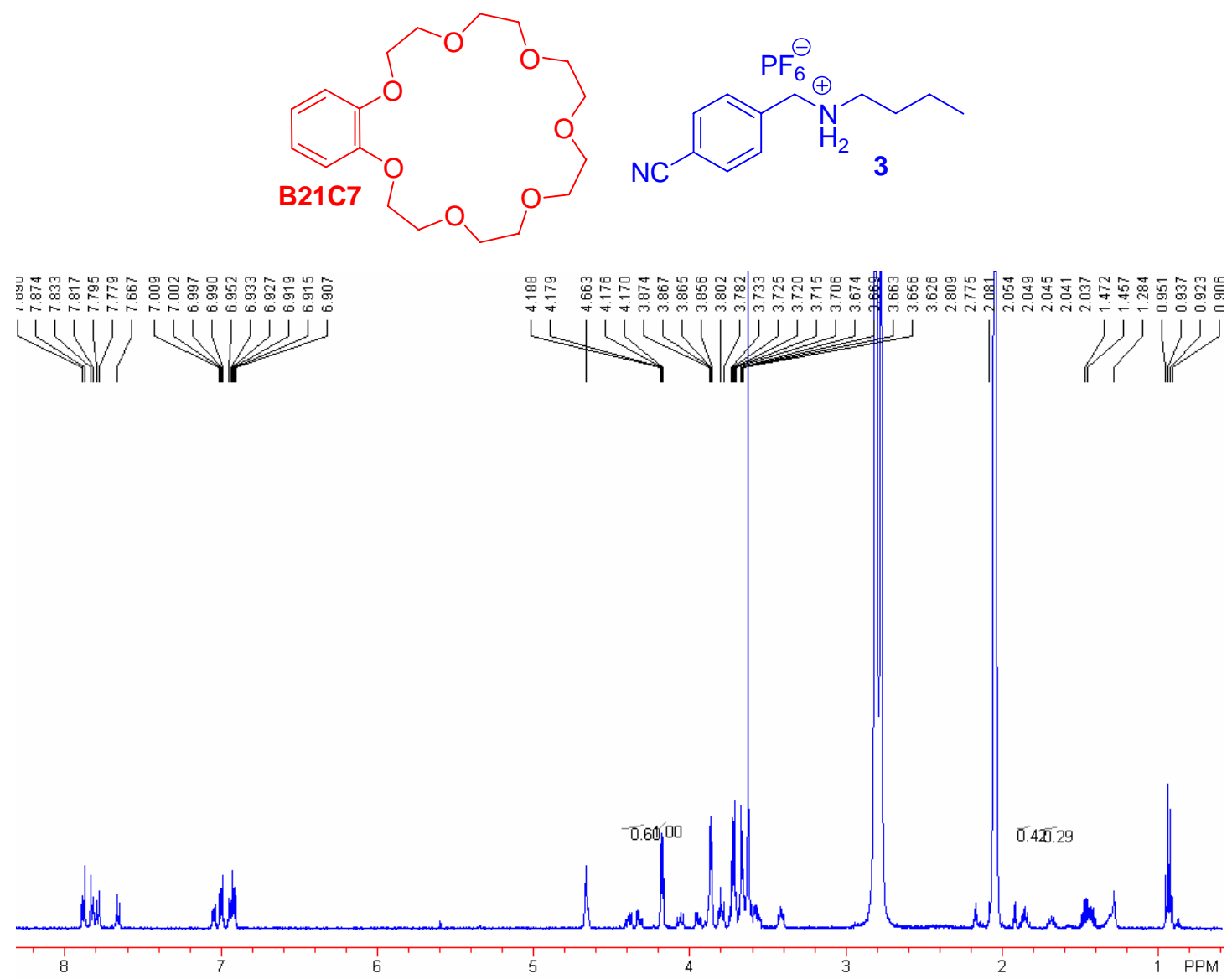

Figure S24. ${ }^{1} \mathrm{H}$ NMR spectrum $\left(500 \mathrm{MHz}, \mathrm{CD}_{3} \mathrm{COCD}_{3}, 22{ }^{\circ} \mathrm{C}\right)$ of $1.00 \mathrm{mM} \mathbf{B 2 1 C 7}$ and 3. The association constant $K_{\mathrm{a}, \mathbf{B 2 1 C 7} \cdot 3}$ value calculated from integrations of complexed and uncomplexed peaks of $\mathrm{H}_{3}$ of B21C7 is $\left[(0.60 / 1.60) \times 1.00 \times 10^{-3}\right] /[(1-0.60 / 1.60) \times 1.00 \times$ $\left.10^{-3}\right]^{2}=960 \mathrm{M}^{-1}$. The association constant $K_{\mathrm{a}, \mathbf{B} 21 \mathrm{C7} \cdot 3}$ value calculated from integrations of complexed and uncomplexed peaks of $\beta$-methylene protons of 3 is $[(0.29 / 0.71) \times 1.00 \times$ $\left.10^{-3}\right] /\left[(1-0.29 / 0.71) \times 1.00 \times 10^{-3}\right]^{2}=1164 \mathrm{M}^{-1}$. Therefore, $K_{\mathrm{a}, \mathbf{B 2 1 C 7} \mathbf{0 3}}=(960+1164) / 2=$ $1062( \pm 102) \mathrm{M}^{-1}$. 


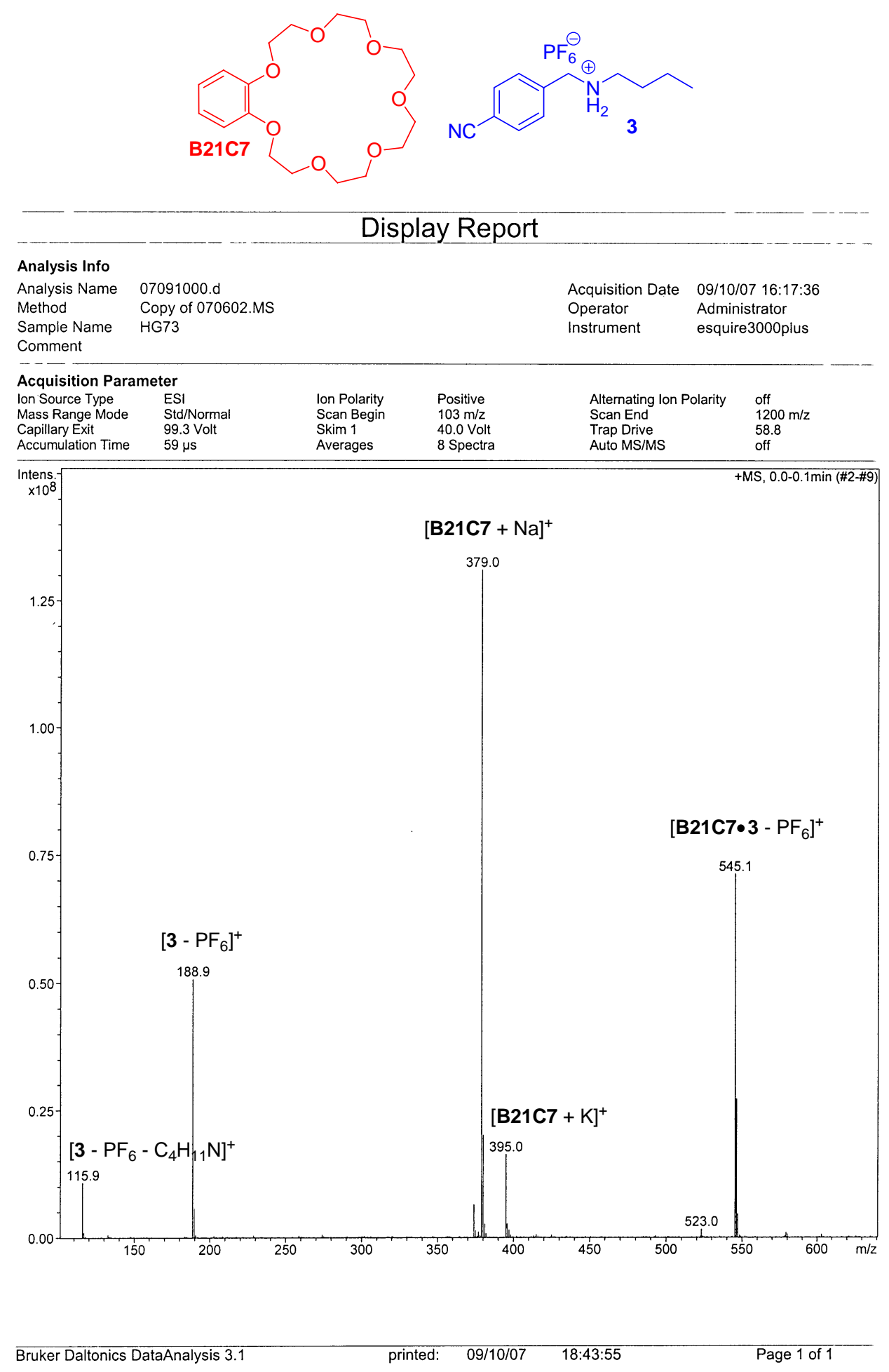

Figure S25. Electrospray ionization mass spectrum of a solution of B21C7 and 3 (1:1 molar ratio). $m / z 115.9(8 \%)\left[3-\mathrm{PF}_{6}-\mathrm{C}_{4} \mathrm{H}_{11} \mathrm{~N}\right]^{+}, 188.9(39 \%)\left[3-\mathrm{PF}_{6}\right]^{+}, 379.0$ $(100 \%)[\mathbf{B} 21 \mathrm{C} 7+\mathrm{Na}]^{+}, 395.0(13 \%)[\mathbf{B} 21 \mathrm{C} 7+\mathrm{K}]^{+}, 545.1(55 \%)\left[\mathbf{B} 21 \mathrm{C} 7 \cdot 3-\mathrm{PF}_{6}\right]^{+}$. 
16. Determination of association constants of DB24C8・1, DB24C8•2, and DB24C8•3 from chemical shift changes.

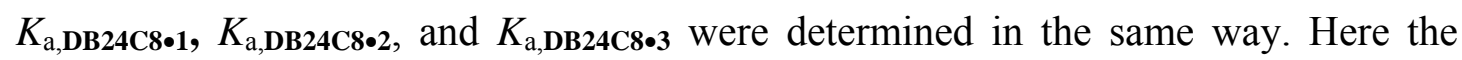
determination of $K_{\mathrm{a}, \mathbf{D B} \mathbf{2 4 C 8} \cdot \mathbf{1}}$ is given as an example. Proton NMR characterizations were done on solutions with constant $[\mathbf{1}]_{0}, 4.00 \mathrm{mM}$, and varied [DB24C8 $]_{0}(8 \sim 25$ $\mathrm{mM}$ ). Based on these proton NMR data, $\Delta_{0,1}$, the difference in $\delta$ values for $\beta$-methylene protons $\left(\mathrm{H}_{10}\right)$ of $\mathbf{1}$ in the uncomplexed and fully complexed species, was determined by the extrapolation of a plot of $\Delta=\delta-\delta_{\mathrm{u}}$ vs. $1 /[\mathbf{D B 2 4 C 8}]_{0}$ in the high initial concentration range of DB24C8. Then $K_{\mathrm{a}, \mathbf{D B} 24 \mathbf{C 8} \cdot 1}$ was calculated from $K_{\mathrm{a}, \mathbf{D B} 24 \mathbf{C} 8 \mathbf{* 1}}=\left(\Delta_{\mathbf{1}} / \Delta_{0, \mathbf{1}}\right) /\left\{1-\left(\Delta_{\mathbf{1}} / \Delta_{0, \mathbf{1}}\right)\right\}\left\{[\mathbf{D B 2 4 C 8}]_{0}-\left(\Delta_{\mathbf{1}} / \Delta_{0,1}\right)[\mathbf{1}]_{0}\right\}$ in an acetone- $d_{6}$ solution of $1.00 \mathrm{mM} \mathrm{DB24C8}$ and 1 . The $\Delta_{0,1}$ value for DB24C8•1 was determined to be $0.242 \mathrm{ppm}$ in acetone- $d_{6}$. The error of $K_{\mathrm{a}, \mathbf{D B} 24 \mathbf{C} 8 \cdot 1}$ is based on the error of $\Delta_{0,1}$ and the initial concentrations of DB24C8 and 1.
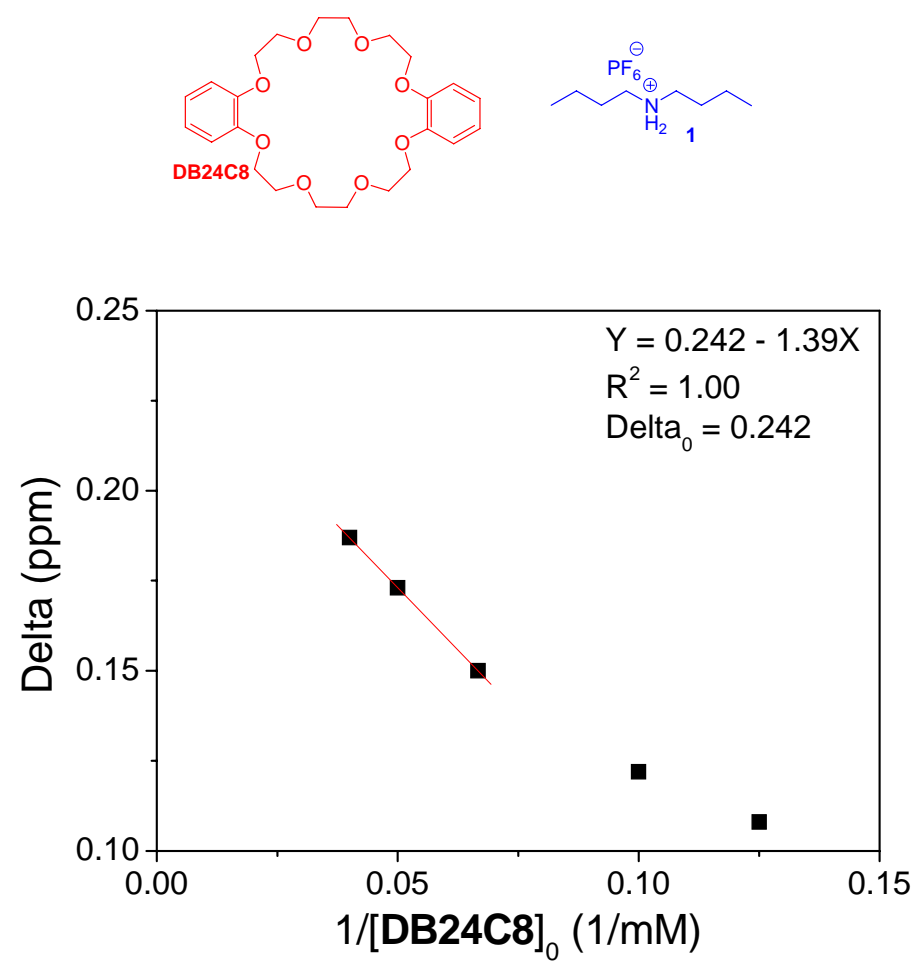

Figure S26. Relationship between $\Delta$ and $1 /[\mathbf{D B 2 4 C 8}]_{0}$ for the complexation between DB24C8 and 1 in acetone- $d_{6}, 22{ }^{\circ} \mathrm{C}$. [DB24C8 $]_{0}$ and $\left[\mathbf{1}_{0}\right.$ are initial concentrations of DB24C8 and 1. [1 $]_{0}$ is constant at $4.00 \mathrm{mM} . K_{\mathrm{a}, \mathbf{D B 2 4 C 8} \bullet \mathbf{1}}=135( \pm 6) \mathrm{M}^{-1}$ at $1.00 \mathrm{mM} \mathrm{DB24C8}$ and $\mathbf{1}$ in acetone- $d_{6}$. 


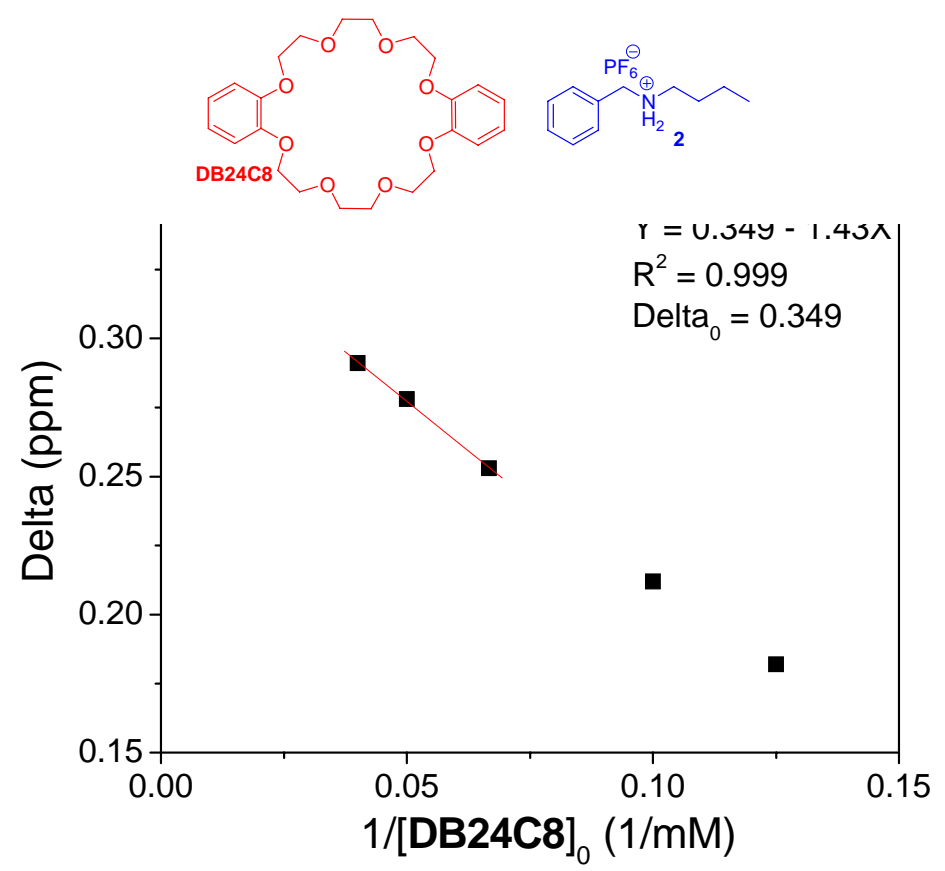

Figure S27. Relationship between $\Delta$ and $1 /[\mathbf{D B 2 4 C 8}]_{0}$ for the complexation between DB24C8 and 2 in acetone- $d_{6}, 22{ }^{\circ} \mathrm{C}$. [DB24C8 $]_{0}$ and $[2]_{0}$ are initial concentrations of DB24C8 and 2. [2 $]_{0}$ is constant at $4.00 \mathrm{mM} . K_{\mathrm{a}, \mathbf{D B 2 4 C 8} \bullet 2}=155( \pm 8) \mathrm{M}^{-1}$ at $1.00 \mathrm{mM} \mathrm{DB24C8}$ and $\mathbf{2}$ in acetone- $d_{6}$.

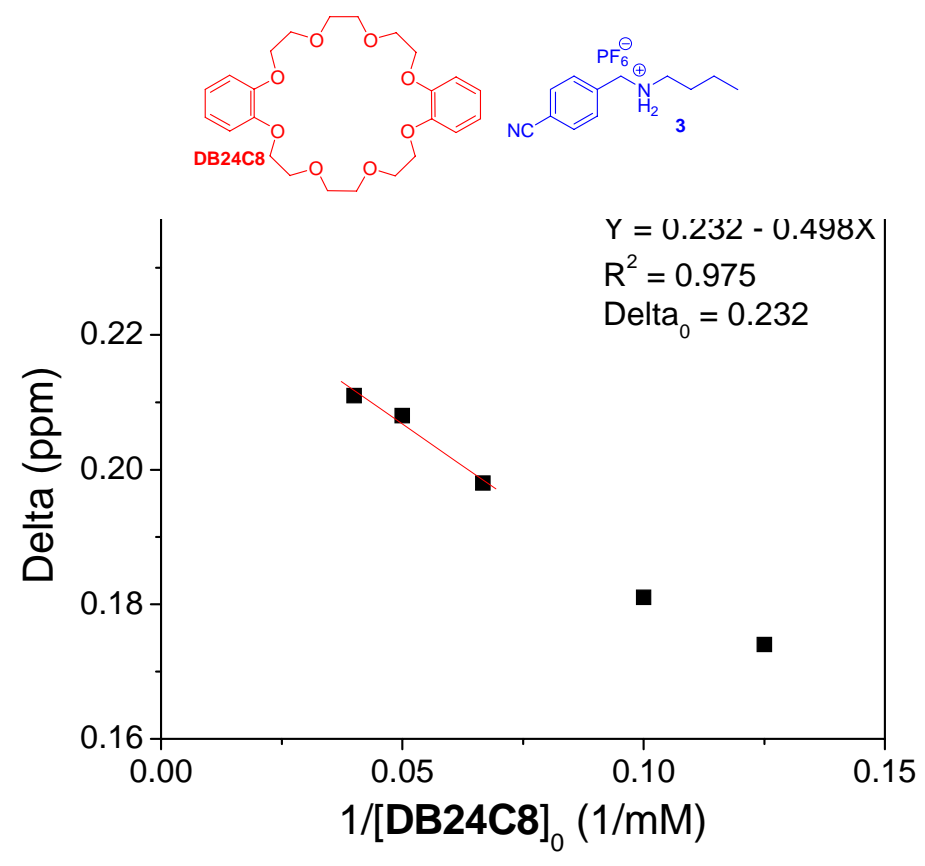

Figure S28. Relationship between $\Delta$ and $1 /[\mathbf{D B 2 4 C 8}]_{0}$ for the complexation between DB24C8 and 3 in acetone- $d_{6}, 22{ }^{\circ} \mathrm{C}$. [DB24C8 $]_{0}$ and $[3]_{0}$ are initial concentrations of DB24C8 and 3. [3] $]_{0}$ is constant at $4.00 \mathrm{mM} . K_{\mathrm{a}, \mathrm{DB} 24 \mathrm{C} 8 \bullet 3}=261( \pm 13) \mathrm{M}^{-1}$ at $1.00 \mathrm{mM}$ DB24C8 and 3 in acetone- $d_{6}$. 


\section{References:}

S1. Tatyana, B.; Stepan, B.; Catherine, K.; Alexander, L.; Nikolay, L. Synthesis 2002, 15, 2266-2270.

S2. (a) Ashton, P. R.; Chrystal, E. J. T.; Glink, P.; Menzer, T. S.; Schiavo, C.; Spencer, N.; Stoddart, J. F.; Tasker, P. A. White, A. J. P.; Williams, D. J. Chem. Eur. J. 1996, 2, 709-728. (b) Cantrill, S. J.; Youn, G. J.; Stoddart, J. F.; Williams, D. J. J. Org. Chem. 2001, 66, 6857-6872.

S3. Stefanie, K.; Matthias, U.; Maike, G.; Wolfgang, W.; Karen, C.; Lan, L. Preparation of 1,2,4-oxadiazol-5-one derivatives as PPAR $\delta$ agonist. PCT Int. Appl. 2007. 